\title{
Excitation Dependent Fano-like Interference Effects in Plasmonic Silver Nanorods
}

\author{
Sean M. Collins, ${ }^{1, *}$ Olivia Nicoletti, ${ }^{1}$ David Rossouw,,${ }^{1,2}$ Tomas Ostasevicius, ${ }^{1}$ and Paul A. Midgley ${ }^{1, \dagger}$ \\ ${ }^{1}$ Department of Materials Science and Metallurgy, University of Cambridge, \\ 27 Charles Babbage Road, Cambridge, CB3 OFS, United Kingdom \\ ${ }^{2}$ Department of Materials Science and Engineering, McMaster University, \\ 1280 Main Street West, Hamilton, ON, L8S 4L7, Canada
}

(Dated: September 4, 2014)

\begin{abstract}
Surface plasmon resonances (SPRs) in metal nanoparticles are an emerging technology platform for nano-optics applications from sensing to solar energy conversion. The electromagnetic near field associated with these resonances arises from modes determined by the shape, size, and composition of the metal nanoparticle. When coupled in the near field, multiple resonant modes can interact to give rise to interference effects offering fine control of both the spectral response and spatial distribution of fields near the particle. Here, we present an examination of experimental electron energy loss spectroscopy (EELS) of silver nanorod monomer surface plasmon modes and present an explanation of observed spatial amplitude modulation of the Fabry-Pérot resonance modes of these silver nanorods using electrodynamics simulations. For these simulations, we identify differences in spectral peak symmetry in light scattering and electron spectroscopies (EELS and cathodoluminescence) and analyze the distinct near field responses of silver nanorods to plane wave light and electron beam excitation in terms of a coupled oscillator model. Effects of properties of the material and the incident field are evaluated, and the spatially resolved EELS signals are shown to provide a signature for assessing Fano-like interference effects in silver nanorods. These findings outline key considerations and challenges for interpreting electron microscopy data on plasmonic nanoparticles for understanding nanoscale optics and for characterization and design of photonic devices.
\end{abstract}

PACS numbers: 79.20.Uv, 73.20.Mf, 78.67.Bf, 78.20.Bh

\section{INTRODUCTION}

Near field interference effects in plasmonic materials represent a promising mechanism for tuning optical responses of metal nanoparticles for ultra-sensitive detection. ${ }^{1}$ Fano resonances commonly give rise to asymmetric and narrow line widths, offering fine control for applications including chemical and biological sensing, ${ }^{2-4}$ nanoscale measurement, ${ }^{5,6}$ and optical switching. ${ }^{7,8}$ While these interference effects are routinely observed in far field light scattering, direct observation of the near field response, particularly with electron beam techniques, often does not offer straightforward interpretation. ${ }^{9-13}$ Here, we present an examination of interfering resonances, or equivalently hybridized modes, in silver nanorod monomers through a combined experimental, simulation, and modeling study of their signatures in scanning transmission electron microscopy electron energy loss spectroscopy (STEM-EELS). In order to understand the experimental EELS results, EELS simulations are compared with simulated cathodoluminescence (CL) and light scattering using discrete dipole approximation (DDA) calculations for both electron and plane wave light excitations. The interference effects observed with STEM-EELS in nanorod monomers can in turn be used to improve the analysis of near field interactions among quasi-one dimensional surface plasmon resonances (SPRs) of metal nanoparticles.

SPRs in nanorods are of particular interest in applications where tunability and polarization sensitivity are critical. ${ }^{14-16}$ Nanorod modes observed in EELS, ${ }^{17-25}$ cathodoluminescence (CL), ${ }^{26,27}$ near field ${ }^{28-32}$ and other measurements ${ }^{33}$ have frequently been described as FabryPérot type resonances, ${ }^{21,22,26,30}$ standing wave patterns of surface charge and the corresponding near fields. These are characterized by intensity at antinodes in the standing wave pattern, recorded as peak intensities in maps and line profiles along the plasmonic rod. FabryPérot resonances have also been reported in metallic strip antennas with film modes determined by the width of the strip, ${ }^{34,35}$ though these differ from rod modes in the azimuthal character of the surface plasmon response and rod modes are reported with azimuthally symmetric charge distributions. ${ }^{16,36,37}$ The multipolar series of longitudinal modes can be labeled by the number of nodes $(m=1,2,3, \ldots)$.

In the simplest case, a Fabry-Pérot resonance exhibits a standing wave pattern determined by an integer number of wavelengths matched to the length of the rod with equal amplitude at each antinode. Additional losses in such a system give rise to a relative phase offset and broadening of the response as a function of driving frequency. Several reports have previously presented experimental near field maps exhibiting spatial modulation of the amplitude at the antinodes along the rod length, particularly in multipolar resonances. ${ }^{21,22,26,28,38,39}$ However, only a few comments have been offered with limited explanation of the observed effect. Qualitative comparisons of light scattered near field distributions, including their spatial amplitude modulation, have been made, requiring the inclusion of multiple plane wave polarizations to reproduce the odd modes in light scattering. ${ }^{22,38}$ Recent theoretical and experimental ${ }^{36,40,41}$ work on plas- 
monic metal nanorods using far field light scattering has demonstrated that multipolar resonances in nanorods interact in the near field, giving rise to Fano-like interferences. We explain the observed amplitude modulation in electron beam driven resonances in terms of the interference phenomena giving rise to Fano-like resonances in far field light scattering. The Fano-like line shape asymmetry of light scattering from rods, reported experimentally ${ }^{36,40}$ and observed in simulations here (Sec. IV) and elsewhere, ${ }^{36,41}$ is not reproduced in EELS spectra of interfering modes and a distinct line shape asymmetry is observed in simulated CL spectra. As shown by analyses of simulated responses to electron excitation, the observed spatial amplitude modulation in EELS and CL corresponds to mode hybridization among electron-driven resonances of the same symmetry (i.e., modes with an odd or even number of nodes, respectively). Moreover, in contrast to light scattering, ${ }^{36}$ electron-excited nanorods exhibit interference effects for both odd and even symmetry modes. This distinction between electron beam and plane wave light excitation identifies that far field signatures of near field interference depend on the incident driving field. The model proposed for understanding the differences in observations in electron spectroscopy and light scattering of silver nanorods represents a general consideration for comparisons of electron- and light-driven plasmonic interference phenomena.

Following an overview of methods in Sec. II, experimental EELS maps, associated spectra, and experimental and simulated line profiles are examined in Sec. III. Section IV presents a comparison of simulated light- and electron-excited spectra and near fields. Observed differences (Sec. IV) are subsequently analyzed by fitting simulations to a coupled oscillator model in Sec. V and the results of the oscillator model are applied to understand the spatial amplitudes of the interfering modes. Parameters that tune the hybridization of the longitudinal modes in silver nanorods are discussed in Sec. VI, a fitting procedure to examine multiple mode interactions is evaluated in Sec. VII, and a summary and conclusions are offered in Sec. VIII.

\section{METHODS}

\section{A. Sample Preparation}

The samples used in this work were acquired from two different sources. For experiments conducted at the University of Cambridge (Rod A in Fig. 1), transmission electron microscope samples of silver nanorods, made by polyol synthesis ${ }^{42}$ (Nano Research Facility, Washington University in St Louis), were prepared by depositing drops of a silver nanoparticle solution on a 30-nm-thick silicon nitride membrane (Agar Scientific). For experiments conducted at McMaster University (Rod B in Fig. 1), samples were prepared as reported previously in Ref.
22.

\section{B. Data Acquisition}

STEM-EELS spectrum imaging was performed on two different FEI Titan electron microscopes. Experiments at the University of Cambridge (Rod A in Fig. 1) were conducted with an FEI Titan ${ }^{3}$ 80-300 equipped with an X-FEG electron source and Wien filter monochromator operated at $300 \mathrm{kV}$. The system energy resolution was recorded at $0.18 \mathrm{eV}$ full width at half maximum (FWHM) in this configuration. Experiments at McMaster University (Rod B in Fig. 1) were conducted as previously reported in Ref. 22, likewise at $300 \mathrm{kV}$ but with a system energy resolution of $0.09 \mathrm{eV}$ FWHM at the time of data acquisition. The large scan size required for the rods leads to a small beam tilt across the field of view if the tilt-shift purity is imperfect. This leads to a small beam "wobble" at the entrance aperture of the spectrometer but this is minimized by a post-specimen beam de-scan. Rod lengths were measured from separately acquired annular dark field micrographs. Rod A was measured at $536 \mathrm{~nm}$ and Rod B was measured at $494 \mathrm{~nm}$. The approximate lengths are given to $10 \mathrm{~nm}$ precision in Fig. 1 and Sec. II as calibration and micrograph measurement error may account for a few percent error in lengths.

\section{Spectral Processing}

Spectrum images were processed using the open-source software HYPERSPY ${ }^{43}$ After spectra were aligned to the zero loss peak (ZLP), the spectrum images were scaled to normalize the Poisson noise ${ }^{44}$ and factorized using a projected non-negative matrix factorization (NMF) algorithm. ${ }^{45}$ EELS maps were divided pixel by pixel by the component corresponding to the ZLP. Similar results were obtained for EELS maps divided pixel by pixel by the zero loss peak intensity recorded in the original spectrum image (see also Supplemental Material (SM) ${ }^{46}$ ).

\section{Simulations}

Discrete dipole approximation (DDA) simulations were performed for electron beam excitation using a customized version of e-DDA ${ }^{47}$ updated for compatibility with DDSCAT7.2 and DDSCAT7.3. ${ }^{48,49}$ DDA simulations were performed for light scattering with DDSCAT7.2. ${ }^{48,49}$ In the DDA formulation, the EELS signal is calculated according to: ${ }^{47,50}$

$$
\Gamma^{\mathrm{EELS}}=\frac{1}{\pi \hbar^{2}} \sum_{j=1}^{N} \operatorname{Im}\left\{\mathbf{E}_{j}^{i n c} \cdot \mathbf{P}_{j}\right\},
$$

where $\mathbf{E}_{j}^{i n c}$ is the incident electric field at dipole position $j$ due to the electron beam, $\mathbf{P}_{j}$ is the polarization of the 
dipole at $j$ due to the incident field and the induced field of the other dipoles, and $N$ is the total number of dipoles.

CL simulations were performed following Ref. 11 where the CL signal is calculated according to:

$$
\Gamma^{\mathrm{CL}}=\frac{c\left|\mathbf{x}_{S}\right|^{2}}{4 \pi^{2} \hbar^{2} \omega} \int d \Omega\left|\mathbf{E}\left(\mathbf{x}_{S}, \omega\right)\right|^{2}
$$

where the position vector $\mathbf{x}_{S}$ determines the radius of the spherical surface over which the integration is performed, $\Omega$ is the solid angle, $c$ is the speed of light in vacuum, and $\hbar \omega$ is the energy of the radiated light. Integration was performed using a custom Python script to integrate the field $\mathbf{E}$ over a sphere of radius $5 \mu \mathrm{m}$ centered on the particle. More than 2,000 points on the spherical surface were included in the numerical integral with even sampling in the azimuth angle and even sampling in $\cos (\theta)$ where $\theta$ is the zenith angle. Convergence was confirmed by also calculating $\Gamma^{\mathrm{CL}}$ for more than 5,000 points and for a sphere at $10 \mu \mathrm{m}$ from the center of the particle. The field $\mathbf{E}$ was calculated using code from e-CL-DDA compatible with DDSCAT7.1. ${ }^{11}$ Rod A was modeled to be $536 \mathrm{~nm}$ long, from tip to tip, with a $17 \mathrm{~nm}$ radius and hemispherical ends each with the same radius as the rod. For near field, near field phase, and line shape analyses (Fig. 7, Fig. 9), Rod A was discretized with $1 \mathrm{~nm}$ dipole spacing, giving a total of 472,781 dipoles. For line profile simulation and dipole moment analysis, rods were discretized with $2 \mathrm{~nm}$ dipole spacing due to the large computational domains required. A comparison of results for different discretization is given in the SM. ${ }^{46}$ Spectral peak positions, near field response, and near field phase were found to be consistent for both $1 \mathrm{~nm}$ and $2 \mathrm{~nm}$ dipole spacings. A finite substrate extending $58 \times 646 \times 30 \mathrm{~nm}$ was used for calculations on Rod A with a substrate. Rod A was modeled with a total of 66,326 dipoles without a substrate and 206,826 dipoles with a substrate. Rod B was modeled to be $494 \mathrm{~nm}$ long with a $13 \mathrm{~nm}$ radius for a total of 36,136 dipoles (2 nm dipole spacing). For simulations without a substrate, an effective purely real ambient dielectric environment was used, a common adjustment ${ }^{51}$ justified for rods where the effect of a substrate primarily redshifts mode energies. The ambient constant refractive index was set to 1.337 for Rod A and to 1.190 for Rod $\mathrm{B}$ to match mode energies to experimental data. These two effective ambient refractive indices reflect the different substrates (silicon nitride and carbon, respectively) in the experimental set-ups for Rod A and Rod B. Dipole moments were calculated following methods outlined in Ref. 52. Dielectric functions were taken from Ref. 53 for silver and Ref. 54 for silicon nitride. For analysis of damping, Drude model dielectric functions were calculated of the form reported in Ref. 55.

Instrumental broadening effects were also taken into consideration, modeled as a convolution of finely stepped simulations (0.01 eV step) with a Gaussian function. In EELS, electrons are emitted from the gun with a finite energy spread, observed in the width of the EELS signature of electrons passing through the sample with little or no energy loss (the ZLP). ${ }^{56}$ This intrinsic width of the ZLP gives rise to an experimental broadening of the energy loss spectrum. ${ }^{56}$ Though the experimental ZLP is not truly Gaussian, the ZLP is often described in terms of its full width at half maximum (FWHM) ${ }^{57,58}$ and a Gaussian function can adequately model the major contributions to ZLP broadening. ${ }^{13}$

\section{E. Fitting Procedures}

As the NMF decomposition describes energydependent peaks associated with the STEM-EELS maps, DDA simulations were performed with fine energy steps over an extended energy range and peak fitting routines were used to extract peak amplitudes for comparison with results from NMF processing of the experimental data. Peak fitting was not used for experimental data as the assumption that the ZLP is approximately Gaussian is particularly inaccurate in describing the ZLP tail (broadening effects described above are not as sensitive to the tail). No ZLP tail is present in simulated spectra and the simulated plasmon resonance peaks were relatively well-described by Lorentzian functions, and so peak fitting was performed for DDA simulations to describe the resonance peaks. Peak fitting of simulated spectra was performed using HYPERSPY. ${ }^{43}$ Peaks were fitted to Lorentzian functions or Voigt functions in cases where ZLP broadening was accounted for by using a convolution of Gaussian and Lorentzian functions (see also SM) ${ }^{46}$ Extended coupled oscillator (ECO) model fitting (Sec. V) was likewise performed using HYPERSPY with model equations following Ref. 51. For ECO model fitting, the dipole moment spectra were first normalized using a single oscillator model for the lowest energy resonance such that the incident field amplitude $E_{0}=1.0$ (units follow Ref. 51). The resonance frequencies $\omega_{1,2}$ were fixed according to fits of the simulated EELS spectra. The resulting ECO model spectra for the decomposed phase response of the interacting modes captured in the displacement amplitude coefficient $\operatorname{Re}\left\{C_{1,2}\right\}$ and far field scattering were calculated using custom scripts in MATLAB. Fitting of Eq. 12 and Eq. 14 was performed in MATLAB using the Curve Fitting Toolbox.

\section{EXPERIMENTAL OBSERVATIONS}

Higher order multipolar longitudinal resonances in silver nanorods exhibiting spatial amplitude modulation appear at visible light energies for rods approximately $500 \mathrm{~nm}$ in length. Figure 1 presents STEM-EELS maps of such modes for a $540 \mathrm{~nm}$ long rod on a $30 \mathrm{~nm}$ silicon nitride membrane substrate $(\operatorname{Rod} A)$ and a $490 \mathrm{~nm}$ long rod on an ultrathin carbon film (Rod B). These maps were prepared by NMF of the experimental data set into two positive matrices, the maps and associated 
spectral components. ${ }^{43,45}$ An NMF decomposition approach was applied recently to EELS measurements of hybridized SPRs in silver nanocubes. ${ }^{12}$ Similar multivariate statistical modal decomposition approaches have also been applied independently to the analysis of modes of silver nanorods ${ }^{38}$ and silver cubes. ${ }^{13}$ Spectral decomposition importantly separates overlapping spectral features and does not presuppose any particular line shape. Notably, the components identified by NMF processing correspond to those already observed in energy-filtered data sets (see in particular Ref. 22 and Fig. 6) but with enhanced signal-to-noise. In EELS, electrons losing negligible or no energy while passing through the sample appear as a ZLP in the energy loss spectrum and give rise to spectral broadening and a spectral tail at low energy losses, including visible light energies. STEM-EELS of Rod A was acquired with a ZLP of approximately 180 meV FWHM whereas for Rod B, STEM-EELS was acquired with a ZLP of approximately $90 \mathrm{meV}$ FWHM. In order to account for differences in the ZLP tail intensity for electrons passing through the silver particle and those passing outside the nanorod, these maps were additionally divided by the component map associated with the tail of the ZLP. To clarify the effects of this procedure, the unprocessed NMF results are included in the SM alongside results obtained by dividing the component maps by the ZLP at each pixel in the original spectrum image. ${ }^{46}$ Accounting for varying ZLP intensity is crucial for reproducing a signal for comparison with simulated energy loss probabilities (see also Ref. 12).

A common feature in all multipolar modes is the low intensities recorded at the rod ends where the energy loss signal (i.e., the EELS probability) is weak because the local fields are predominantly perpendicular to the electron trajectory. The EELS probability, in contrast, is related to the component of the field parallel to the trajectory as given by: ${ }^{59}$

$$
\Gamma^{\mathrm{EELS}}=\frac{q}{\pi \hbar \omega} \int d t \operatorname{Re}\left\{e^{i \omega t} \mathbf{v} \cdot \mathbf{E}^{i n d}\right\},
$$

where the integral is with respect to time $t, q$ is the charge on the electron, $\hbar \omega$ is the energy loss in terms of angular frequency $\omega$, and $\mathbf{v}$ and $\mathbf{E}^{\text {ind }}$ are the electron velocity and induced electric field vectors, respectively. The notation and units follow Ref. 59.

The spectral signatures associated with each of the maps in Fig. 1 are presented in Fig. 2 for a single electron trajectory at the rod tip (4 nm offset) where all longitudinal resonances are excited. The transverse (trans) and bulk excitations are very weakly excited for the selected trajectory. Fig. 2 demonstrates that each of the components maps in Fig. 1 correspond to separate peaks in the spectrum. These peaks exhibit a dominant amplitude at the particular energies noted in Fig. 1. Moreover, the unprocessed data (blue dots) are well reconstructed by the NMF decomposition but with significantly reduced noise (black solid line). The effect of the ZLP is pronounced in Fig. 2 as the peaks are barely visible as modulations

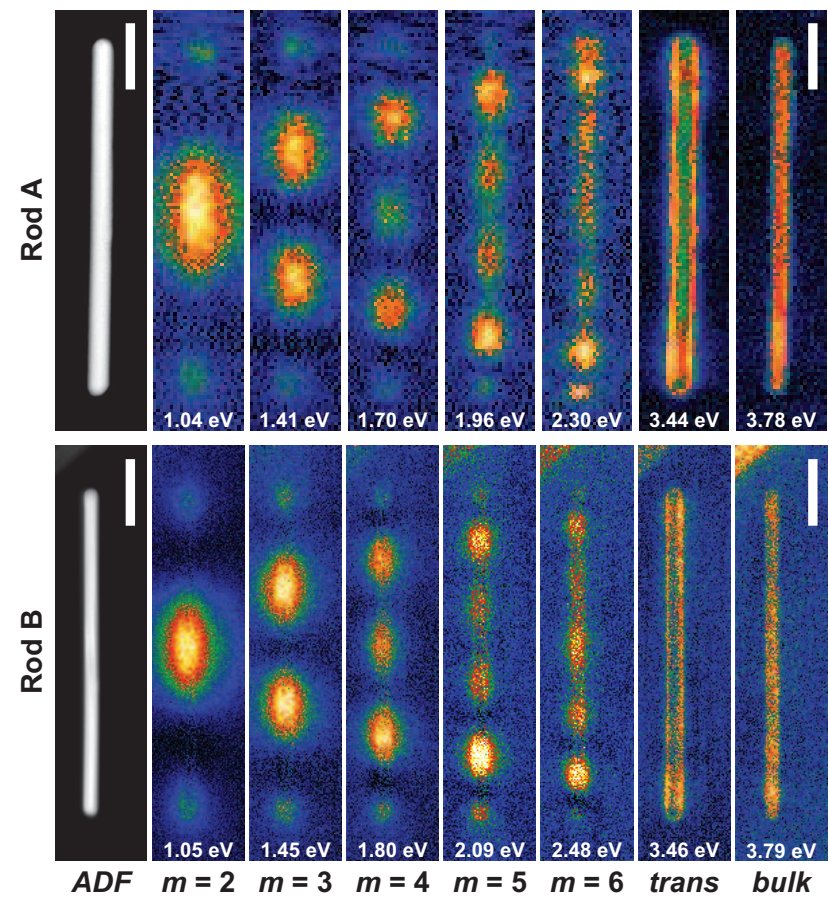

FIG. 1. (Color online) STEM-EELS maps for two silver nanorods approximately $540 \mathrm{~nm}(\operatorname{Rod} A)$ and $490 \mathrm{~nm}(\operatorname{Rod}$ B) in length. Longitudinal modes $m=2-6$ are shown as well as transverse (trans) and bulk excitations. Annular dark field (ADF) micrographs are shown for reference. Rod A was prepared on a $30 \mathrm{~nm}$ thick silicon nitride membrane and Rod B was prepared on an ultrathin carbon substrate (Reprocessed from Ref. 22). Maps were extracted by non-negative matrix factorization (NMF) techniques. Scale bars are $100 \mathrm{~nm}$.

of the ZLP tail particularly for Rod A acquired with a broader ZLP than for Rod B. The NMF decomposition approach makes use of the entire spectrum image and accounts for the Poissonian noise present in the data allowing for feature extraction even at pixels where the signals are weak.

For direct comparison of the experimentally observed plasmon resonance modes with simulations in Fig. 3-4 and in subsequent sections, it is critical that the experimental data is processed to determine the energy loss probability. Such processing requires the separation of the ZLP and division by the varying ZLP intensity as noted for Fig. 1. The NMF decomposition approach achieves this necessary separation with minimal bias and without assumptions on the shape of the ZLP or resonance peaks. Alternative approaches such as peak fitting and ZLP subtraction ${ }^{22,60}$ make significant assumptions about the spectral shape of the ZLP and of the resonance peaks.

The ZLP shape does not match perfectly with what might be expected for a smoothly varying ZLP tail, and in particular minor modulations of the spectral dependence at or close to energies coinciding with surface plasmon peaks are visible in the ZLP components in Fig. 2. 

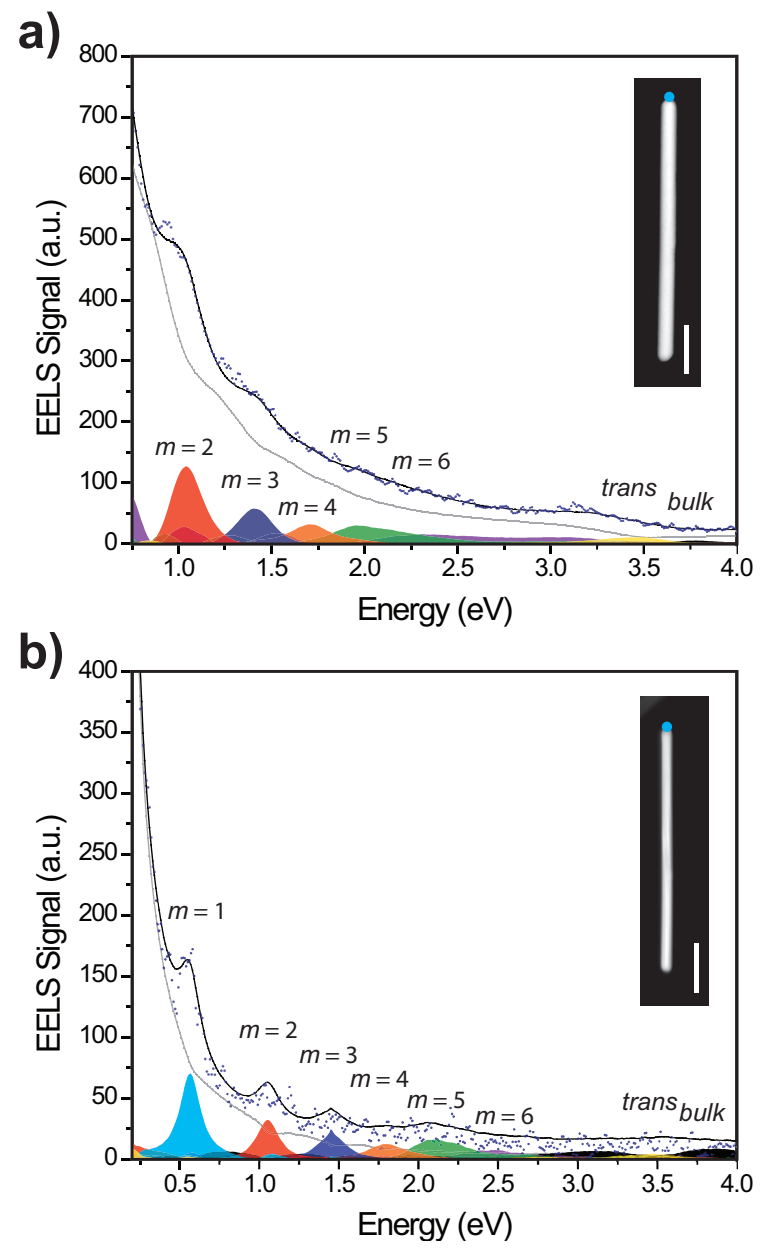

FIG. 2. (Color online) NMF spectral components for (a) Rod $\mathrm{A}$ and (b) Rod B weighted according to the value for a single pixel in the STEM-EELS maps in Fig. 1 at the position indicated by the blue marker in the inset $(4 \mathrm{~nm}$ offset from rod tip, see also Fig. 3). The unprocessed spectrum (blue dots) is shown together with the spectral components for the zero loss peak (gray solid line), the longitudinal resonances $m=2-6(\operatorname{Rod} \mathrm{A})$ and $m=1-6(\operatorname{Rod} \mathrm{B})$, transverse (trans) mode excitations, and the silver bulk plasmon. The sum of the spectral components is also presented (solid black line). Scale bars are $100 \mathrm{~nm}$.

These modulations can be attributed to the numerical nature of the NMF appraoch and represent minor deviations from an ideal ZLP at these energy channels. The magnitudes of the modulations are small relative to both the spectral intensity of the surface plasmon peaks and the ZLP. In the context of the intensity maps of each of these components, taking into account the entire peak, these imperfections in the decomposition will contribute very little to the mapped EELS probability, contributing to the background in the EELS maps due to residual intensity from the ZLP (Fig. 3-4). The intensity modulation in particular is not affected as the spectral components are fixed for all pixels in the map and it is the modal surface plasmon peak amplitude divided by the a)
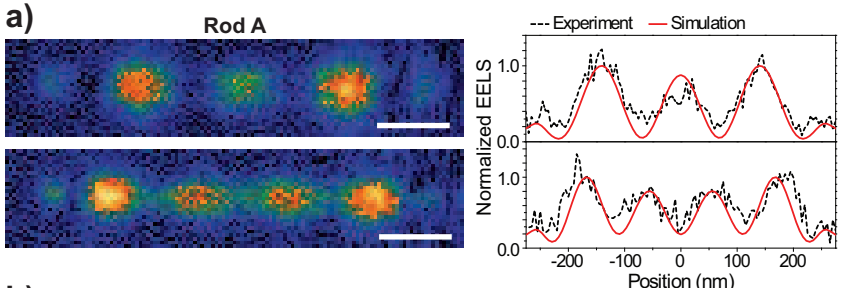

b)
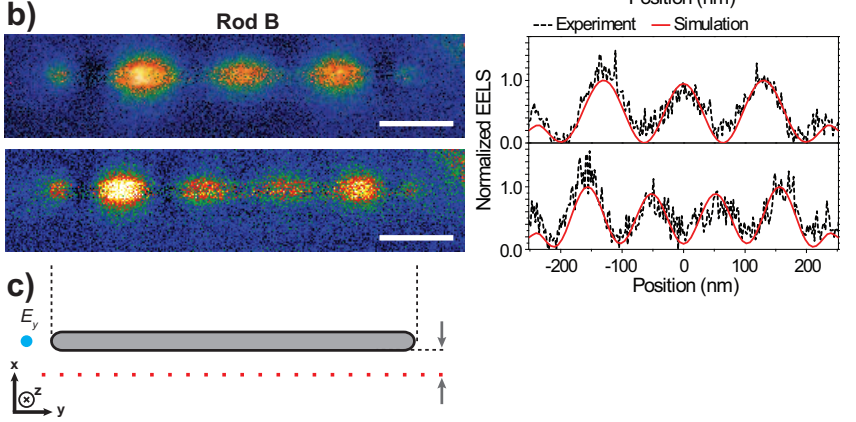

FIG. 3. (Color online) Comparison of experimental and simulated line profiles. (a)-(b) STEM-EELS maps and line profiles ( $4 \mathrm{~nm}$ from rod side) of modal components $m=4$ and 5 for (a) Rod A and (b) Rod B. Extracted experimental line profiles are compared with simulated line profiles calculated by DDA methods. Simulations on Rod A included a $30 \mathrm{~nm}$ thick silicon nitride membrane. Simulations on Rod B were performed using an effective ambient medium due to uncertainties in the dielectric properties of the experimental carbon membrane grid. (c) The coordinate system, positions of the line profiles, and coordinate selected for phase analysis $\left(E_{y}\right)$ are shown for reference. Scale bars are $100 \mathrm{~nm}$.

\section{ZLP amplitude plotted in Fig. 3 and 4.}

Figure 3 presents maps for modes $m=4$ and 5 and corresponding line profiles ( $4 \mathrm{~nm}$ from the rod side) from experimental data and from discrete dipole approximation (DDA) simulations ${ }^{47-49}$ for an incident electron with 300 keV primary energy. Modes $m=4$ and 5 are the lowest order resonances where amplitude modulation is observable. For these modes, the intensity at antinodes in the energy loss map decreases towards the center of the rod. This is not a characteristic expected for a Fabry-Pérot resonance with equal amplitude throughout the standing wave. This amplitude modulation has been documented elsewhere and is observed in rods of varying lengths. ${ }^{21}$

The DDA simulated line profiles in Fig. 3 match the experimental profiles well, reproducing the same observed spatial amplitude modulation. For Rod A, DDA simulations were performed including a finite silicon nitride substrate. For Rod B, an effective ambient dielectric medium was used to model the ultrathin carbon film substrate as in other nanorod studies ${ }^{51}$ (see also $\mathrm{SM}^{46}$ ). By comparing two similar rods on different substrates and examined with different instruments, the experimental data and simulations indicate that the observed amplitude modulation is not uniquely determined by the composition of the substrate or particular properties of the data acquisition (e.g., microscope energy resolution). 
a)

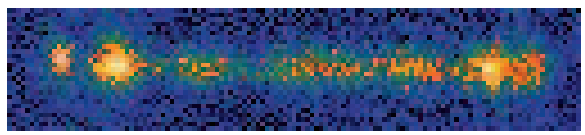

b)

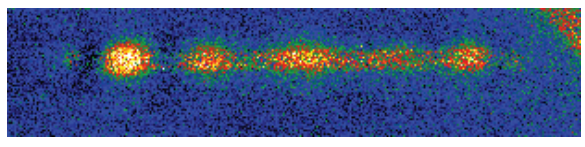

c)

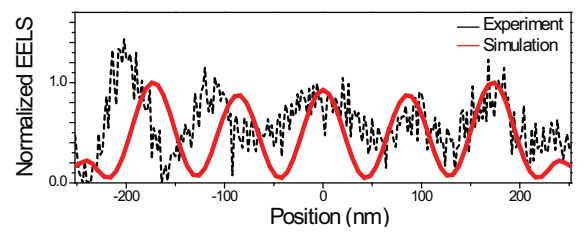

FIG. 4. (Color online) STEM-EELS maps of mode $m=6$ for (a) Rod A and (b) Rod B and (c) the corresponding line experimental and simulated line profiles for Rod B.

Further simulations describing the effects of the substrate and experimental energy resolution are included in the SM. ${ }^{46}$

Figure 4 presents a comparison of the $m=6$ mode for Rod A and Rod B. Mode $m=6$ was at the noise level for Rod A and no meaningful line profile could be extracted for trajectories at $4 \mathrm{~nm}$ offset from the side of the rod. For Rod B, the spatial intensity maps and line profiles for $m=6$ identify a more subtle modulation of intensity along the rod axis, specifically the local intensity maximum visible at the center of Rod B for mode $m=6$ (Fig. $4(\mathrm{~b})(\mathrm{c}))$.

Rod B exhibits a partial spatial asymmetry in the intensity pattern for mode $m=6$, as previously noted in initial reports on this rod..$^{22}$ The dielectric environment was not constant for this sample as the lacey carbon support visible in the upper right corner (Fig. 3(b), Fig. 4(b)) demonstrates a local modification, expected to impact more significantly the more sensitive higher order modes. ${ }^{55}$ Because the experimental intensities at antinodes (intensity maxima) were systematically brighter for the left hand side of Rod B for all modes, the simulated line profiles were vertically scaled to the consistent intensities at the center and right hand side of the rod in the experimental data. Fig. 4(b) highlights the experimental observation of a local maximum of EELS intensity at the center of the rod with a global maximum near the ends. That is, in the EELS map, the antinode at the center appears brighter than the two immediately adjacent. This observation is consistent with the DDA simulations (Fig. $4(\mathrm{c}))$. The DDA simulation of the line profile does not appear to match as well as for modes $m=4$ and $m=6$. However, the point of comparison is the relative intensity of the antinodes; the differences between the DDA simulated line profile and the experimentally extracted line profile are (1) a translation offset and (2) an asymmetric intensity distribution from left to right. The spatial amplitude modulation pattern in the experiment is in fact quite similar to the simulated line profile in that the cen- tral antinode is brighter than the two adjacent antinodes.

The spectral signatures (Fig. 2) associated with each of the mode maps presented in Fig. 1 and Fig. 3-4 provide additional information about the energy dependence of the modes. Figure 5 presents the individual NMF spectral factors for modes $m=4$ and $m=5$ for Rod A and $m=4-6$ for Rod B. The peaks for mode $m=4$ are quite symmetric. The spectral factors for modes $m=5$ and $m=6$ exhibit asymmetric tails at higher energies as well as minor "satellite" peaks at lower energies. These "satellite" peaks do not correspond to additional resonance mode signatures at lower energies, but contribute to a small background intensity (Fig. 2) attributed to imperfect numerical separation by NMF processing. From the NMF decomposition alone it is not possible to determine whether the spectral line shapes purely represent physical effects or are the result of incomplete separation of overlapping spectral features. From our experience of applying NMF to EELS datasets, we find in general that the algorithm produces spatial distribution maps with few artifacts but spectral factors that exhibit side peaks and imperfect line shapes. Previous analyses of these and other rods indicate highly symmetric peak shapes for summed spectra across the entire spectrum image ${ }^{22}$ or from selected areas of the spectrum image. ${ }^{21}$

To further clarify the effects of NMF processing, maps extracted from the STEM-EELS data cube with an energy window of $0.02 \mathrm{eV}$ near the $\mathrm{m}=5$ resonance are presented for Rod A in Fig. 6. The ZLP was first aligned at each pixel, but otherwise no processing was performed on these energy-filtered maps. The overall intensity decreases from $1.92 \mathrm{eV}$ to $2.00 \mathrm{eV}$ due to the decreasing background intensity of the ZLP. The signal distribution along the rod at these energies is otherwise very similar to $m=5$ recovered in NMF processing. These maps have not been divided by the ZLP intensity and so the intensities inside the rod are low. The consistency of the amplitude modulation observed in the experimental data acquired on Rod A for energies below and above the resonance energy at $1.96 \mathrm{eV}$ is also consistent with DDA simulations which exhibit symmetric peaks (see also Sec. IV).

The consistent spatial amplitude modulation observed in NMF decomposition (Fig. 1), energy-filtered maps (Fig. 6, and reported previously for Rod $\mathrm{B}^{22}$ and for other rods ${ }^{21}$ in energy-filtered maps indicates the spatial amplitude modulation is intrinsic to the EELS response of silver rods and is not introduced by processing methods. In fact, the NMF decomposition demonstrates that the effect is not simply due to spectral overlap of modes as the decomposition substantially reduces such overlap relative to energy-filtered mapping. Ultimately, the close correspondence between the experimental and the DDA simulated line profiles establishes that the DDA simulations describe the experimental system well, enabling additional investigation of the simulated responses in Sec. IV-VI.

The experimental data presented here describe a se- 
a)

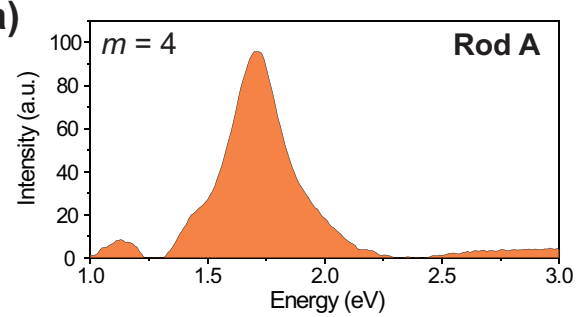

b)

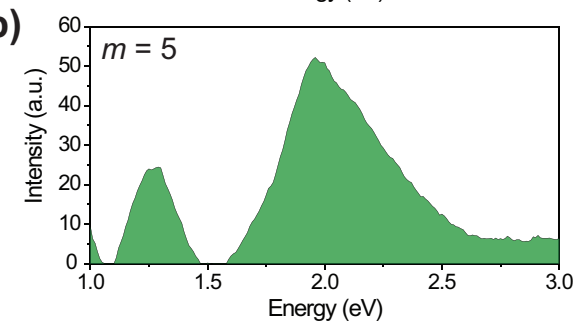

c)

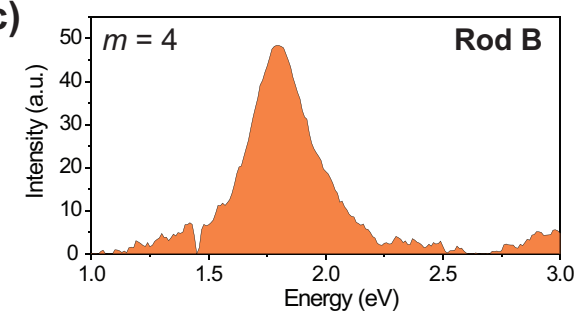

d)

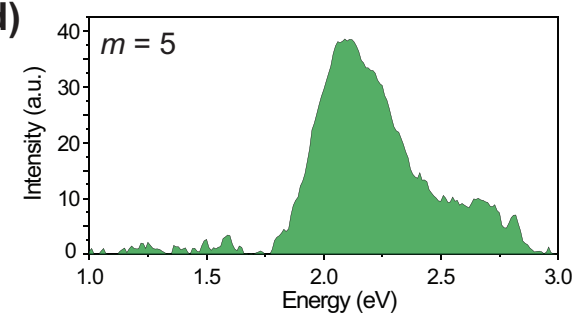

e)

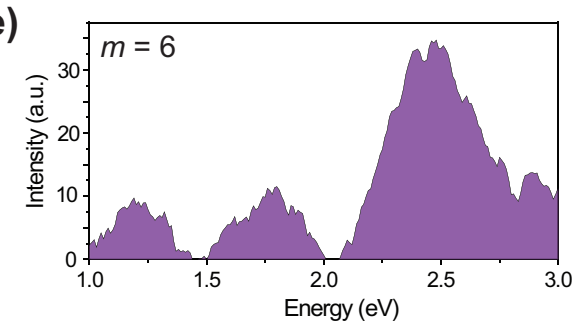

FIG. 5. (Color online) Extracted NMF spectral factors for modes $m=4$ and $5(\operatorname{Rod} \mathrm{A})$ and $m=4-6(\operatorname{Rod} \mathrm{B})$.

ries of Fabry-Pérot-like modes that, however, exhibit an unexpected spatial amplitude modulation. This spatial amplitude modulation is consistently observed across the resonance in the energy spectrum (Fig. 5-6). These observations prompt a more detailed analysis of the simulated electron excitations to understand the origins of the spatial amplitude modulation and to examine the reported Fano-like resonance (equivalently, the mode hybridization effects) expected in such nanorods. ${ }^{41}$

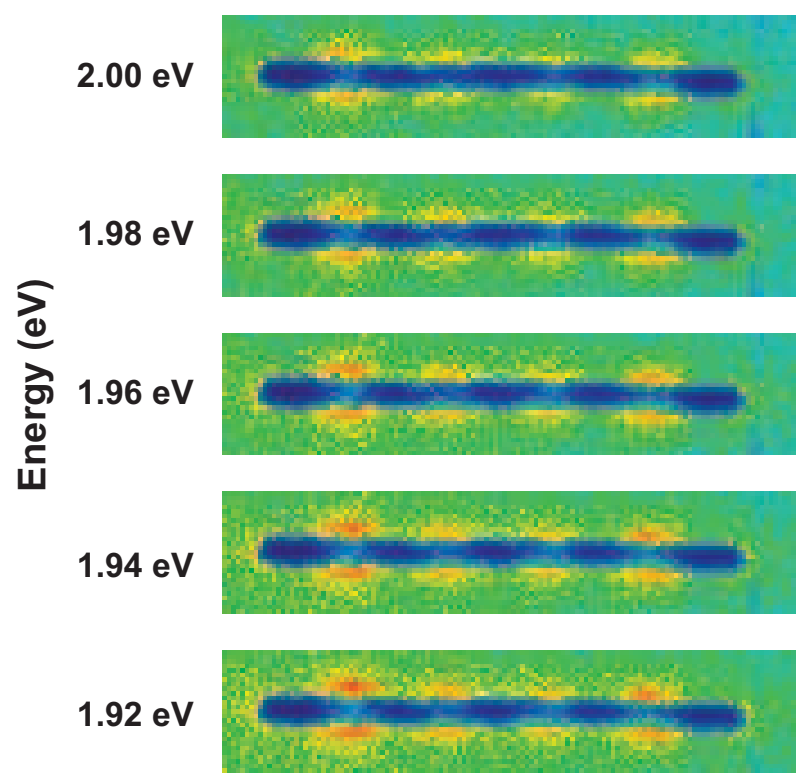

FIG. 6. (Color online) Images filtered around $0.02 \mathrm{eV}$ windows extracted from the STEM-EELS data cube for Rod A at energies in the vicinity of the $m=5$ longitudinal resonance. The color table is on a single scale for all energies.

\section{COMPARISON OF SIMULATED LIGHT SCATTERING, EELS, AND CL SPECTRA}

Fano-like interference effects give rise to spectral signatures commonly in the form of asymmetric peaks due to destructive and constructive interference effects in the near field. This section compares spectra for light and electron excitation and the associated near fields. The simulations presented here build on experimental results and simulations reported previously for light scattering ${ }^{36,40}$ and $\mathrm{CL}^{26}$ of similar plasmonic nanorods. These DDA simulations, validated by comparison with experiment in Sec. III, serve to identify similarities and differences in spectral and near field responses to light and electron beam excitation prompted by apparent contradictions in the experimental results to date. Along with the subsequent sections, these simulations present a comprehensive computational analysis of the origin of the observed experimental amplitude modulation in EELS.

In light scattering, the interference of longitudinal resonances in silver nanorods has recently been explored theoretically ${ }^{41}$ and observed experimentally. ${ }^{36,40}$ Modes are often classified as "bright" for odd modes exhibiting antisymmetric character about the central mirror plane of a rod and "dark" for even modes exhibiting symmetry about the same mirror plane. ${ }^{19}$ In light scattering, odd modes interact in the near field with each other and a dipolar continuum due to the illuminating field giving rise to Fano-like resonances. ${ }^{36,41}$ Figure $7(\mathrm{a}),(\mathrm{c})$ present DDA light scattering simulations for Rod A without a substrate with a single incident electric field polarization parallel to the long axis of the rod. The asymmetric far 
field scattering profile in Fig. 7(c) is consistent with a Fano-like resonance. The "bright" and "dark" modes of a nanorod are distinguished by their symmetry about the central mirror plane perpendicular to the rod axis, and the symmetry is not modified by such factors as the substrate when the symmetry of the rod changes from $D_{\infty h}$ to $C_{2 v}$. The substrate in this system does not play a symmetry breaking role, and so the Fano-like resonance effect is present without a substrate.

Fano-like near field interference effects, or alternately mode hybridization, requires an examination of the relative phases of the interacting modes. ${ }^{13}$ In this section, we first examine the spectral line shape, phase, and near field amplitude as a function of energy for light and electron excitation of these silver nanorods. The relative phase relationships are subsequently decomposed in Sec. $\mathrm{V}$. Here, the phase of the surface plasmon is taken as the phase of the scattered near field component $E_{y}$ parallel to the axis of the rod. The phase of $E_{y}$ is plotted for a coordinate in the plane containing the long axis of the nanorod, offset from the tip by $4 \mathrm{~nm}$ as for the line profiles in Fig. 3, the same position as the electron trajectory in electron simulations. When the surface plasmon charge density wave is in phase with the incident plane wave polarization, the phase of $E_{y}$ is approximately 0 radians (dashed line). The surface plasmon charge density wave is completely out of phase at $\pi$ radians. The phase of $E_{y}$ is approximately $\pi / 2$ at the resonance energy. In Fig. 7(a),(c), the surface plasmon charge density wave is in phase with the incident plane wave at energies preceding the resonance and is out of phase for energies above resonance. The far field absorption spectrum is also plotted for reference in Fig. 7(a). Absorption is an incoherent effect and so does not track with the phase of the surface plasmon response, but provides a marker of the resonance energies. ${ }^{61}$

The response of the silver nanorod to far field light excitation, typical of Fano resonant systems, ${ }^{1}$ can be understood in terms of the response of coupled oscillators. ${ }^{62}$ It is a general property of a single oscillator that it will respond in phase with a driving force at frequencies (energies) below its resonant frequency, and it will respond out of phase with the force when it is driven past its resonant frequency ${ }^{62,63}$ In a coupled oscillator system, an additional case arises in which the lower frequency oscillator is out of phase at frequencies that drive the higher frequency oscillator in phase. Consequently, at frequencies for which the two oscillators are out of phase relative to each other, destructive interference results. For frequencies exceeding the resonance of the higher frequency oscillator, both oscillators are out of phase with the incident plane wave and in phase with each other, giving rise to constructive interference. The transition from destructive to constructive interference gives rise to an asymmetric spectral line shape. This description applies to classical Fano resonance systems observed in numerous plasmonic particle ensembles ${ }^{10,51}$ and in some isolated particles such as a cube on a substrate. ${ }^{55}$
The oscillator model serves to explain the observations in the light scattering simulation, but this description seemingly does not explain the observed EELS response presented in Fig. 7(c) as the EELS response exhibits relatively symmetric peak shapes. As in light scattering (Fig. 7(a)), for electron excitation (Fig. 7(b)) the phase (dashed line) is ca. 0 radians preceding resonances and rises across the resonances. This similar phase behavior indicates that the electron-driven surface plasmon exhibits similar phase response characteristics as the surface plasmon driven by far field light excitation. Additional peaks are observed in the EELS case as both "bright" and "dark" modes are excited by the electron beam. ${ }^{21,22}$ In the EELS case depicted in Fig. 7(b), the phase response differs from Fig. 7(a) in that the phase remains less than $\pi / 2$ radians and so $\operatorname{Re}\left\{E_{y}\right\}$ does not change sign. This lack of a change in the sign of $\operatorname{Re}\left\{E_{y}\right\}$, suggesting incomplete phase inversion, is a local response phenomenon in EELS and has been discussed in previous reports as local phase pinning. ${ }^{10,11}$ When the trajectory is displaced further from the rod $(22 \mathrm{~nm})$, phase changes greater than $\pi / 2$ radians are observed, but the EELS peaks remain symmetric $\left(\right.$ see $\left.\mathrm{SM}^{46}\right)$. The phase inversion character of the electron-driven surface plasmon is typical of an oscillator model, but the expected destructive and constructive interference effects are not readily observed in the spectral EELS signal.

Given that the observation of a Fano line shape can depend strongly on how the response of the system is recorded (i.e., $Q_{a b s}$ v. $Q_{s c a}$ in light scattering), additional simulations were carried out for electron excitation with detection in the far field (CL). CL measures the electromagnetic fields radiated into the far field due to electron beam excitation. A simulated CL spectrum for the same incident trajectory $4 \mathrm{~nm}$ from the rod tip is presented in Fig. 7(d). The CL signal in fact does exhibit an asymmetric line shape (highlighted by blue arrows in Fig. 7(d), see also Sec. VI and discussion of Fano parameter fitting for CL spectra), not apparent in the EELS signal. Comparisons can be drawn between the EELS signal and the extinction cross-section and between CL and the light scattering cross-section. ${ }^{64}$ In the DDA formalism, the EELS signal is calculated as the extinction of the incident field of the electron at a particular frequency. ${ }^{47,50}$ The extinction cross-section is determined from the summation of the absorption and scattering components, ${ }^{48}$ and so the symmetry of the EELS peak relative to the CL peak can be attributed to the relatively high contribution of dissipative (absorptive) losses in the silver nanorod compared to radiative (scattering) losses captured in the CL signal. The asymmetry in the CL signal confirms that a Fano-like resonance effect is present in electron beam excitation similar to that recorded in light scattering. By energy conservation, any electron-induced CL signal corresponds to an energy loss signal as EELS includes both radiative and non-radiative energy losses in the system. The electron excitation and the EELS signal therefore include features of a similar mode hy- 
a)

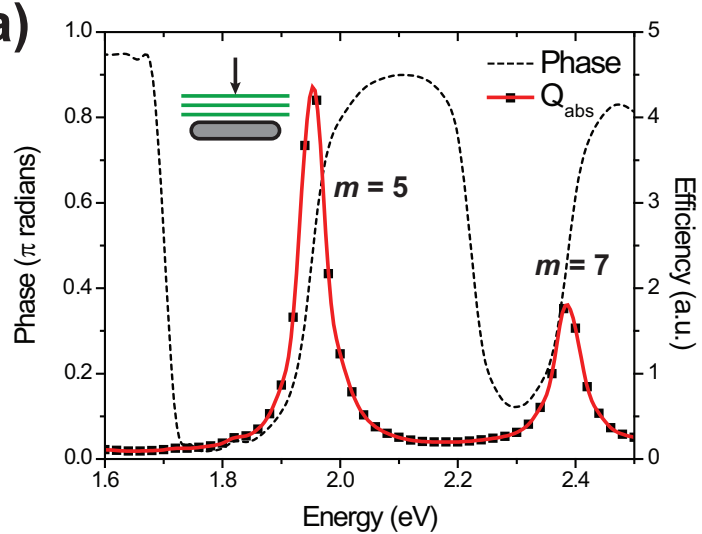

c)

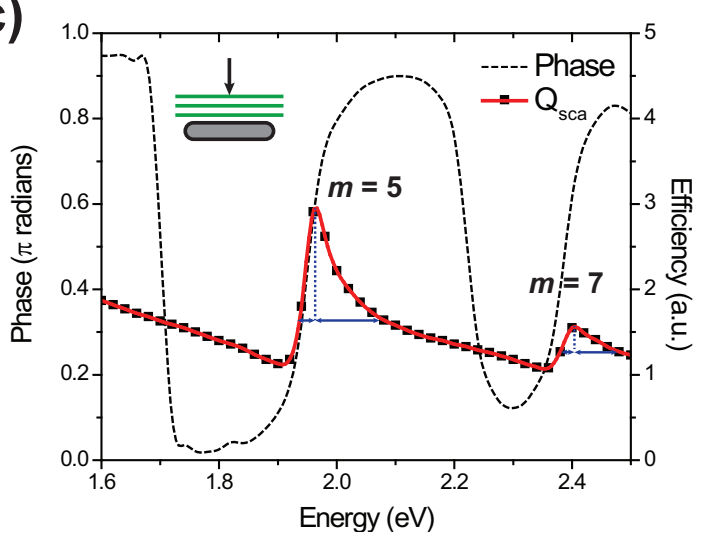

b)

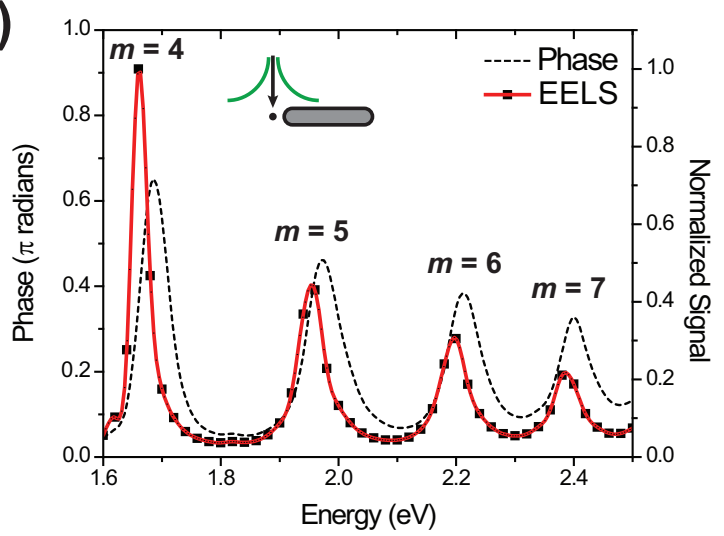

d)

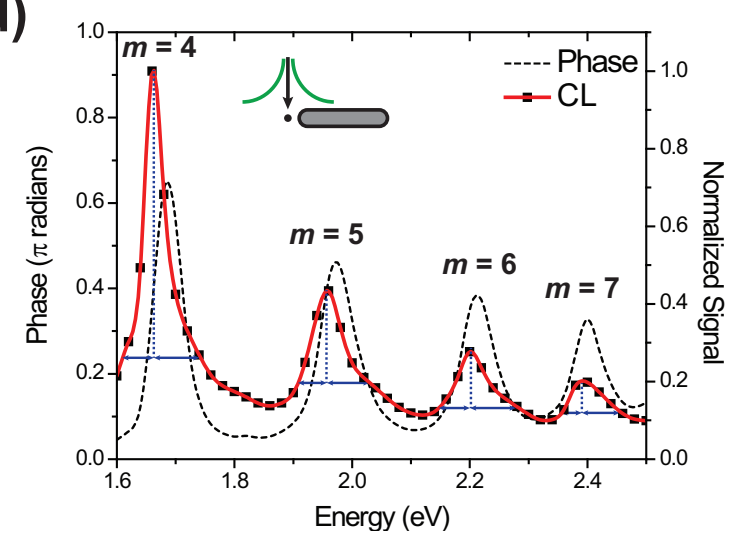

FIG. 7. (Color online) Spectra and phase relationships for (a),(c) plane wave light and (b), (d) electron beam excitation for Rod A (no substrate) simulated by DDA. (a) The light absorption $\left(Q_{a b s}\right)$ and (c) scattering $\left(Q_{s c a}\right)$ efficiencies are plotted for plane wave light excitation. (b) The EELS and (d) CL responses are plotted for electron beam excitation. The electron beam was positioned $4 \mathrm{~nm}$ from the tip of the rod, and the phase here refers to the phase of the induced field along the axis of the rod (y-axis) at the same offset as the electron trajectory and even with the xy-mirror plane (point marked $E_{y}$ in Fig. 3). Black squares denote simulated data points connected by a cubic spline (red solid line).

bridization and near field interference effect, though the spectral signature is not readily observable in EELS.

However, the CL signal differs in key ways from the light scattering spectrum (Fig. 8(a)). Specifically, the low-energy onset of the CL peak is more gradual, exhibiting a positive slope at lower energies, than in light scattering. The light scattering peak exhibits a relatively sharp onset as expected for pronounced destructive interference effects at energies just below the resonance energy. Different mode hybridization is required to account for the onset as the transition from destructive to constructive interference occurs at a lower energy in CL than in the light scattering spectrum. Furthermore, the CL spectrum exhibits slight asymmetry in peaks for both odd and even modes, in contrast to recent light scattering experiments and simulations. ${ }^{36}$ The asymmetry in the CL signal confirms that the electron beam excites coupled modes, and that mode coupling, though producing a distinct spectral response compared to light scattering, is present in electron excitation.

Verellen et al. have recently reported that light excitation results in no interference for even modes due to a symmetry mismatch with the plane wave-induced dipolar continuum background..$^{36}$ For electron excitation, however, the illuminating field is not dipolar, and consequently detection of the interaction between series of modes of even and odd symmetry is possible as identified in the simulated CL spectra. Modal interaction is still restricted by symmetry to modes of the same symmetry (i.e., $m=1,3,5, \ldots$ and $m=2,4,6, \ldots$ can interact) as near field interference effects are linked to spatial and spectral overlap ${ }^{41}$ and modes of different symmetry exhibit minimal spatial overlap and, like a sum of sine and cosine functions, ${ }^{36}$ will not exhibit spatial amplitude "beating" modulation. It is, moreover, a similar symmetry mis-match with the incident dipolar field of plane wave excitation that precludes the detection of mode hybridization and associated inteference phenomena in the far field in light scattering. ${ }^{36}$ Mode hybridization in the near field between even modes is in principle similar to hybridization of odd modes, and the asymmetric far field spectra for electron excitation captured in the simulated CL spectra confirm this effect. These observations encourage additional investigation of the hybridization of 

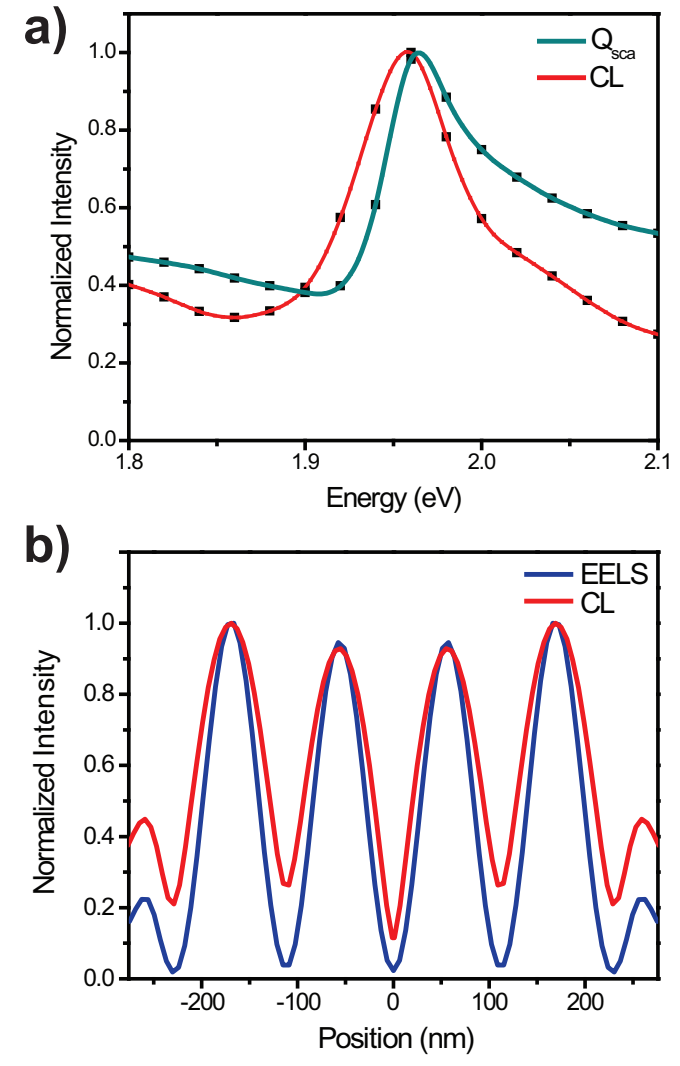

FIG. 8. (Color online) Simulated (a) CL and $Q_{\text {sca }}$ spectra for Rod A. The electron beam was positioned $4 \mathrm{~nm}$ from the tip of the rod (point marked Ey in Fig. 2). Black squares depict simulated data points connected by a cubic spline (solid lines). (b) Simulated EELS and CL line profile for Rod A (no substrate) at $1.98 \mathrm{eV}(m=5)$ and $4 \mathrm{~nm}$ from the side of the rod.

even modes and their associated near fields when driven by plane wave light excitation. Such studies are beyond the scope of this examination of electron-driven modes where simulations do exhibit asymmetric line shapes in the far field (CL spectra) indicative of mode hybridization and Fano-like interference effects for both even and odd modes in silver nanorods.

The different responses of the silver nanorod recorded for electron beam and light excitation, including the consistency of the CL spectral asymmetry for both odd and even modes and the lower energy onset of radiative processes detected by CL, suggests a significant difference in the underlying near field interaction of interfering modes and their detection in the far field. These differences contrast with reports identifying similarities in EELS and light scattering of Fano-resonant cubes $^{13}$ and present a distinct case from multi-particle systems where the spatial separation of interacting modes allows for unique interference effects due to the electron position. ${ }^{11}$

The simulated CL signal also exhibits nearly identical amplitude modulation in a line profile along the rod
(Fig. 8(b)). The ends of the rod give rise to more signal in CL than EELS, consistent with the relative insensitivity of EELS to the fields at the rod tips which are predominantly perpendicular to the electron trajectory whereas CL signals record the fields radiated into the far field in all directions. In both cases, there is also a geometric effect in the line profiles in that the straight-line selection of trajectories for the line profile (Fig. 3) results in greater displacements from the rod surface in the line profile near the tip. Consequently, the low signal at the ends of the line profile is also due to the greater distance from the electron trajectory to the rod surface at trajectories in the vicinity of the rod tip than for trajectories along the rod side. As a purely radiative signal, the amplitude modulation observed in CL, and its close correspondence to that in EELS (Fig. 8(b)), indicates the spatial amplitude modulation is independent of the particular detection scheme and is likely related to the spatial near field distribution of the mode or modes excited in the rod. Experimental work as well as light scattering and CL simulations on similar silver nanorods have been reported previously by Gómez-Medina et al. ${ }^{26}$ Here, we employ CL simulations as a tool to further analyze the electron-excited response of the silver nanorods examined with EELS in Sec. III to understand the radiative and non-radiative contributions to the EELS signal. The work by Gómez-Medina et al. corroborates the simulations in Fig. 7-8, likewise presenting both the asymmetric line shape and the spatial amplitude modulation in CL. ${ }^{26}$ Section V presents additional analyses to understand the origin of these effects due to the hybridization of rod modes.

For the case of odd modes, direct comparison of the respective near field responses to plane wave light and electron excitation is possible. The light- and electroninduced near fields at three energies spanning the $m=5$ resonance are presented in Figure 9. The energies 1.90, 1.96, and $2.02 \mathrm{eV}$ were selected to correspond to energies just below, on, and above the resonance energy at 1.96 $\mathrm{eV}$. Changes in the near field amplitude distribution are apparent across the resonance energy.

The field amplitude at the tips in all cases is relatively weak in Fig. 9 as the field is plotted at a plane above the rod, and the curvature at the rod ends results in a greater distance to the plane at the tips. For plane wave light excitation (Fig. 9, left), white circles emphasize where the near field intensity at the tips changes across the Fanolike resonance from $1.90 \mathrm{eV}$ to $2.02 \mathrm{eV}$. The near field is symmetric from one end of the rod to the other. At $1.90 \mathrm{eV}$, there is destructive interference, resulting in a pronounced decrease in the near field intensity at the rod tips. At $2.02 \mathrm{eV}$, there is constructive interference and enhanced signal at the rod tips. In contrast, for electron beam excitation, white circles indicate similar relative near field intensities above and below the resonance energy, but appearing on opposite ends of the rods for fields above and below the resonance energy. The switch in location of this near field intensity can be attributed to 

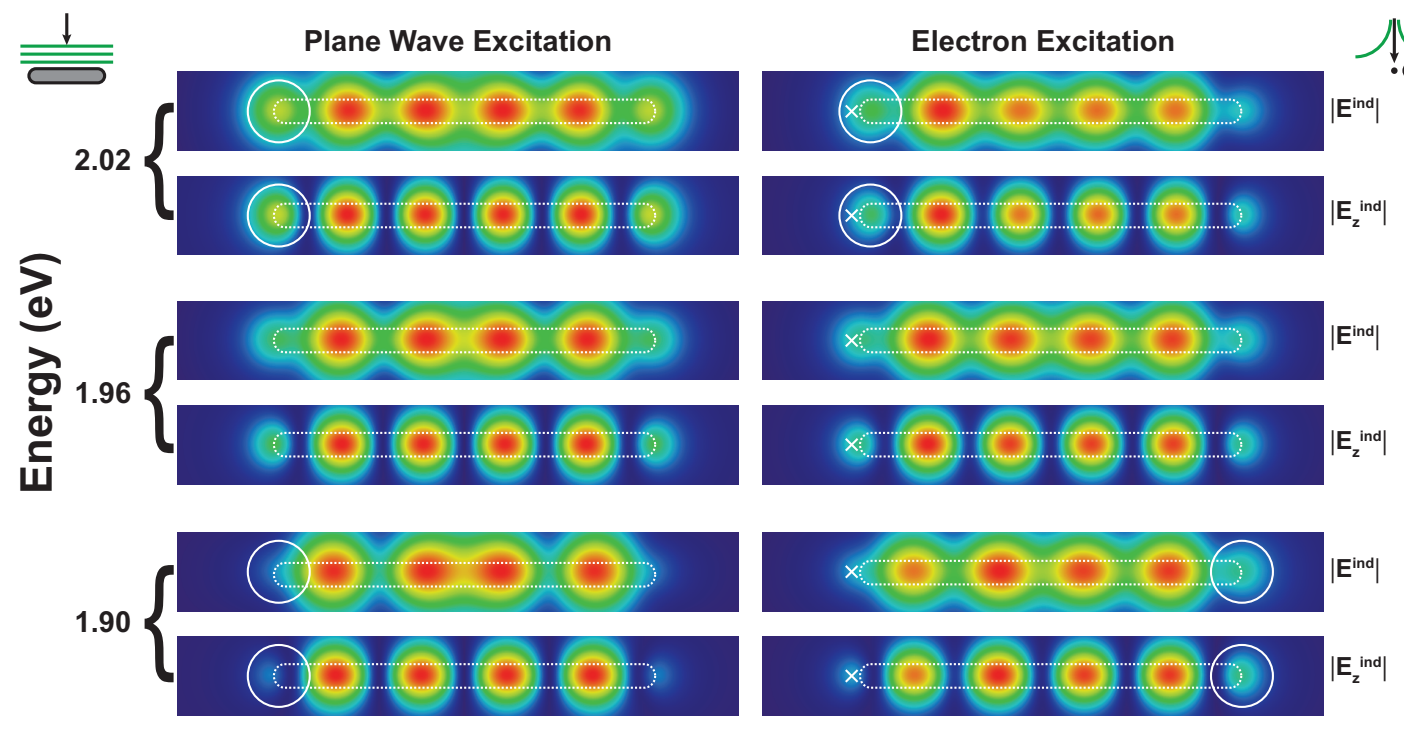

FIG. 9. (Color online) Near field distributions in a plane $40 \mathrm{~nm}$ above Rod A (no substrate) for (left) plane wave light and (right) electron beam excitation. White crosses mark the electron beam trajectory. The total field $\left|\mathbf{E}^{\text {ind }}\right|$ and $\mathrm{z}$-component of the field $\left|\mathbf{E}_{z}^{\text {ind }}\right|$ are shown for each energy. White circles indicate key points of comparison at 1.90 and $2.02 \mathrm{eV}$. Intensities were re-scaled for each image to highlight relative intensities at each energy. The rod shape is illustrated by a dotted white outline.

the noted phase changes in Fig. 7. The electron trajectory is $4 \mathrm{~nm}$ from the left tip in these near field plots. For the electron beam excitation, asymmetry from left to right appears due to the decay of the driving field as a function of distance from the electron trajectory. In Fig. 9, the scattered field is plotted; in the case of the electron beam the incident field exhibits high intensity at the trajectory (marked with a cross). At $1.90 \mathrm{eV}$, the total field is strong at the tip near the electron trajectory and the scattered field is strong at the opposite end where the incident field is weaker. At $2.02 \mathrm{eV}$, the phase flips to opposing the incident field of the electron and the scattered field is therefore intense at the electron beam trajectory acting back on the incident field. The energydependence of the near field is dissimilar to that for plane wave light excitation as there is no clear damping of the net dipole moment. At 1.90 and $2.02 \mathrm{eV}$, there is no apparent cancellation of the electron-induced fields at the rod tips. In contrast, for light excitation the field amplitude at the rod tips is negligible at $1.90 \mathrm{eV}$, consistent with the destructive interference visible at $1.90 \mathrm{eV}$ in the plane wave excited near field.

Direct interpretation of the near field response to electron beam excitation is not straight-forward given two contributions to the spatial amplitude: (1) the incident field decay as a function of distance from the electron trajectory and (2) the induced polarization acting back on the particle. In DDA terms, there are contributions to the total amplitude modulation in the electron excitation case from the spatial modulation of the incident field and from the induced (scattered) electric field. Here, only the scattered field is presented, but its response depends on the incident driving field. It is possible to compare maps of the EELS signal with light scattered fields because an EELS map can be related to potentials in the vicinity of a particle ${ }^{65}$ or a photonic local density of states, at least for systems that exhibit approximate two-dimensionality and translational invariance along the electron trajectory. ${ }^{66}$ The comparison of induced near fields presented here suggests a distinct near field response of the nanorod to the two excitations and begins to illustrate differences in the spectral signals. These difference are explained more fully in terms of the dipole moment along the rod in Sec. V.

\section{EXTENDED COUPLED OSCILLATOR (ECO) MODELING}

The near field response can be captured more quantitatively by examining the net dipole moment along the rod axis. The dipole moment represents the response of the system to excitation separately from a particular detection scheme. This section serves (1) to identify differences in the rod response to light and electron excitation in order to explain the noted differences in spectral line shape in light scattering, CL, and EELS (Sec. IV) by decomposition of the total response into the contributions of the interacting longitudinal modes of the silver rod and (2) to assess the expected spatial amplitude modulation for the hybridized modes observed in electron excitation.

Figure 10(a)-(b) presents the net dipole moment along the rod axis (y-axis) as a function of energy for both light scattering and electron beam excitation for the first two odd modes, $m=1$ and $m=3$. The relative polarization of the $m=3$ mode stands out as a pronounced difference. For light scattering, the $m=3$ mode is only weakly polarized. The difference in relative polarization 
is even more striking at the $m=5$ resonance (Fig. 11(a)(b)). Moreover, for light excitation the dipole moment approaches zero just before the $m=5$ resonance. This energy dependence for light excitation is again consistent with destructive interference below the resonance energy and less radiation into the far field at energies below resonance. For electron beam excitation, the $m=5$ mode is more strongly polarized and exhibits a nearly equal positive dipole moment below the resonance energy and negative dipole moment above the resonance energy (Fig. 11(b)). These differences in the energy-dependent net dipole moment specifically underscore that the near field interactions between the series of longitudinal modes in the nanorod differ depending on the excitation. The distinct relative excitation of modes for light and electron excitation is consistent with previous work identifying different relative weighting factors for surface plasmon modes of metal nanospheres ${ }^{67}$ though the analysis here examines different relative polarizations for coupled modes.

Reconciliation of these differences for light and electron excitation, and the relatively more symmetric line shape in CL relative to $Q_{s c a}$, is possible by considering the ECO model for Fano resonances in systems where both modes are driven by an external force. ${ }^{51,63}$ Coupled oscillator modeling is an analogous approach to other strategies for decomposing the relative phase contributions of the modes including methods such as projecting the polarization response onto a vector basis as recently proposed by Iberi et al. ${ }^{13}$ Coupled oscillator modeling is particularly well-suited to the case of the rod as no expectation of the vector basis is required and the fitting results can be directly related to an expected scattering spectrum. Once the relative phases of interacting modes are determined, a simple Fabry-Pérot model for the spatial distribution can be applied, similar to the Fano-resonance analysis using oscillator modeling and spatial field distributions reported by Lovera et al. ${ }^{51}$

Figure 10(c) depicts this model for a two-oscillator system, each with a resonant frequency $\omega_{1,2}$ coupled by a spring system descbribed by the constant $g .{ }^{51}$ For a twooscillator system, the equations of motion can be written in terms of the normal modes of the system as: ${ }^{51}$

$$
\begin{aligned}
& \ddot{x}_{1}+\gamma_{1} \dot{x}_{1}+\omega_{1}^{2} x_{1}+g x_{2}=0.5 \dddot{P}_{\text {tot }}+\alpha_{1} E_{\text {ext }} \\
& \ddot{x}_{2}+\gamma_{2} \dot{x}_{2}+\omega_{2}^{2} x_{2}+g x_{1}=0.5 \dddot{P}_{\text {tot }}+\alpha_{2} E_{\text {ext }},
\end{aligned}
$$

where dot notation is used to represent first, second, and third derivatives with respect to time, $x_{1,2}$ are the displacements in normal coordinates (i.e. the sum and difference of the displacements of the individual oscillators), $\gamma_{1,2}$ are damping parameters, $\alpha_{1,2}$ are the polarizabilities, and $E_{\text {ext }}$ is the driving field. Subscripts denote each of the two oscillators. The total dipole moment, $P_{t o t}$ is given as: ${ }^{51}$

$$
P_{t o t}=P_{1}+P_{2}=\alpha_{1} x_{1}+\alpha_{2} x_{2},
$$

For a driving force of the form $E_{\text {ext }}=E_{0} e^{i \omega t}$, solutions exist of the form $x_{1,2}=C_{1,2}(\omega) e^{i \omega t} .{ }^{51}$ The complex co-
TABLE I. Fitting parameters for ECO modelling of light scattering and EELS $(m=1, m=3)$. Relative units are indicated in terms of energy units (eV) following Ref. 51.

\begin{tabular}{ccc}
\hline \hline Parameter & $\begin{array}{c}\text { Light Scattering } \\
(m=1, m=3)\end{array}$ & $\begin{array}{c}\text { EELS } \\
(m=1, m=3)\end{array}$ \\
\hline$E_{0}$ & 1.0 & 1.0 \\
$\omega_{1}[\mathrm{eV}]$ & 0.450 & 0.450 \\
$\omega_{2}[\mathrm{eV}]$ & 1.34 & 1.34 \\
$g\left[\mathrm{eV}^{2}\right]$ & $-4.41 \times 10^{-4}$ & $-9.29 \times 10^{-4}$ \\
$\gamma_{1}[\mathrm{eV}]$ & 0.0293 & 0.0291 \\
$\gamma_{2}[\mathrm{eV}]$ & $-4.41 \times 10^{-3}$ & -0.0401 \\
$\alpha_{1}\left[\mathrm{eV}^{-1}\right]$ & 0.153 & 0.153 \\
$\alpha_{2}\left[\mathrm{eV}^{-1}\right]$ & 0.0531 & 0.0914 \\
\hline \hline
\end{tabular}

efficients $C_{1,2}$ are calculated from Eq. 4, and the signs of the real parts of $C_{1,2}$ indicate whether the oscillator modes are in or out of phase at a particular energy. ${ }^{51}$ Notation and units are adopted here from Ref. 51.

This model resembles the case of the nanorod where sequential modes of the same symmetry are driven by either light or a passing electron. In this work, the total dipole moment rather than the scattering spectrum was fitted to the ECO model. Eq. 5 was therefore fitted to the net dipole moment as shown in Fig. 10(a)-(b). To fit the equations for a two-oscillator model, the lowest energy interacting modes were first selected for comparison $(m=1, m=3$ ) (Fig. 10). The two-oscillator model is best suited to the first two modes of the series since the ECO model describes systems where the lower energy resonance is not already coupled to a separate oscillator. For higher energy modes, the lower energy mode interaction may not be negligible (e.g., for $m=3$ and $m=5$, $m=1$ may have a significant effect on $m=3)$.

Figure 10 shows the best fits of Eq. $5\left(P_{t o t}\right)$ to the simulated dipole moments as well as the corresponding energy-dependent coefficients $C_{1,2}$ and the calculated scattering spectrum $\left|C_{1}+C_{2}\right|^{2}$. ${ }^{51,63}$ The corresponding fit parameters are presented in Table I. Units in Table I follow the convention in Ref. 51. In accordance with the noted higher relative polarization of the $m=3$ resonance for electron excitation, the polarizability is nearly twice that for light excitation at $m=3$. The associated damping parameter also changes slightly, and the magnitude of the coupling constant $g$ increases. The negative sign for damping can be attributed to the formulation of Eq. 4 in normal coordinates; the underlying damping parameters of the individual oscillators are physically positive, but the combined damping parameter of the normal coordinate, taken as the difference of the oscillator positions, allows for negative values. The coupling constant $g$ can also be negative as reported previously, ${ }^{51}$ suggestive of repulsive interactions. The signs for these parameters for light scattering and electron excitation are the same, indicating consistency between the two excitations.

Specifically, negative $\gamma$ values (Table I) do not imme- 
a)

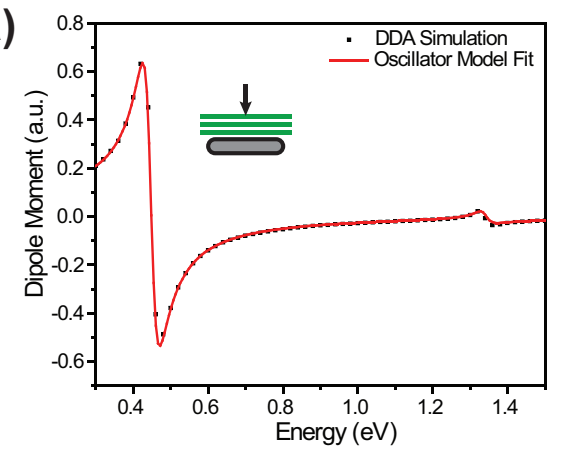

b)

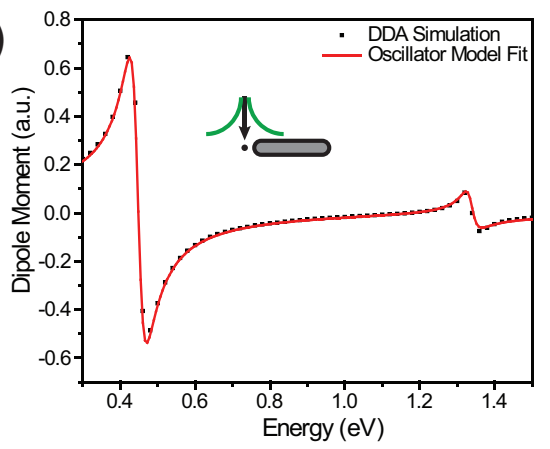

c)

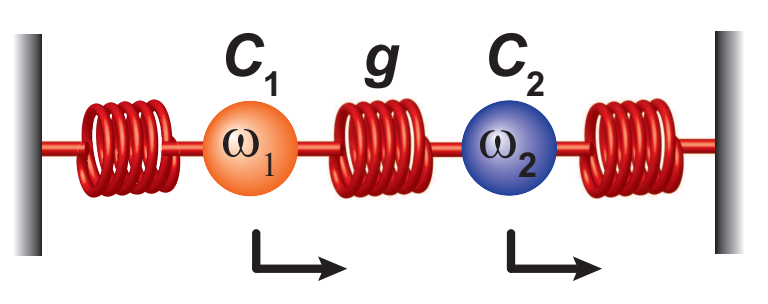

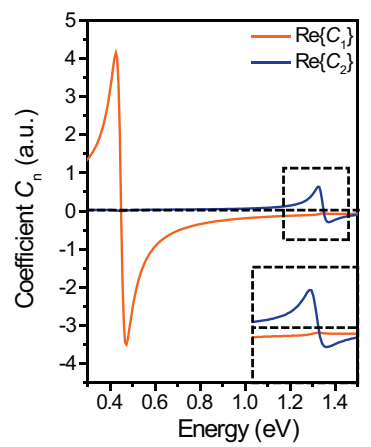
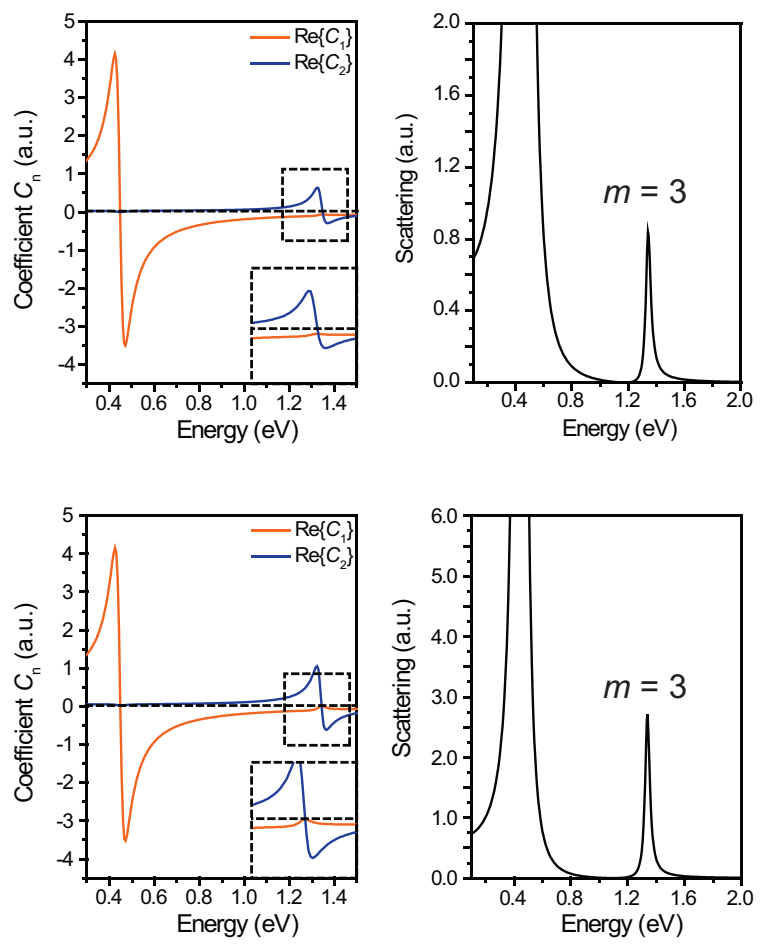

d)

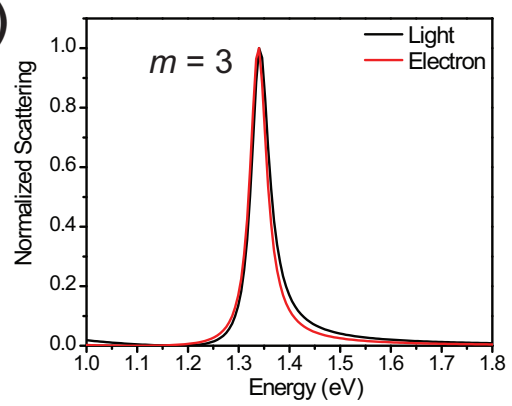

FIG. 10. (Color online) ECO model fitting of the dipole moment data from DDA simulations for Rod A. In each left panel, the dipole moment $P_{t o t}$ along the y-axis of the rod is plotted as well as best fit net dipole moment $P_{t o t}$ for the ECO model (Eq. 5). In the middle panels, the real coefficients for the two oscillators are plotted, and the signs of the coefficients are highlighted at the higher energy resonance. The right panels show the corresponding scattering spectrum calculated from the ECO model $\left|C_{1}+C_{2}\right|^{2}$ for: (a) Light scattering $(m=1, m=3)$, (b) EELS $(m=1, m=3)$. (c) Illustration of the two oscillator model with resonant frequencies $\omega_{1,2}$. The oscillators are coupled by a set of springs described by the constant $g$ (Eq. 5 is written in normal coordinates), the coefficients $C_{1,2}$ describe the displacement and phase of the oscillators, and the arrows indicate the driving force acting on each oscillator. (d) Comparison of scattering line shape at the $m=3$ resonance.

diately appear to be physical. However, when the equations of motion are written in normal coordinates as for the ECO model $\left(x_{1}=z_{1}+z_{2}\right.$ and $x_{2}=z_{1}-z_{2}$ where $z_{n}$ is the displacement of the individual oscillators), the damping constants likewise become defined in terms of the sum and difference of the oscillators, specifically the sum and difference of the first derivatives:

$$
\begin{aligned}
\gamma_{1} & =\frac{\Gamma_{1} \dot{x}_{1}+\Gamma_{2} \dot{x}_{2}}{\dot{x}_{1}+\dot{x}_{2}} \\
\gamma_{2} & =\frac{\Gamma_{1} \dot{x}_{1}-\Gamma_{2} \dot{x}_{2}}{\dot{x}_{1}-\dot{x}_{2}},
\end{aligned}
$$

where $\Gamma_{n}$ here refer to the internal damping parameters for the individual oscillators. Consequently, $\gamma_{2}$ will be negative for small $\Gamma_{2}$ and $\dot{x}_{2}>\dot{x}_{1}$ or large $\Gamma_{2}$ and $\dot{x}_{2}<\dot{x}_{1}$. Negative values are physically possible in this formulation. Further, $\gamma_{1}$ was allowed to remain negative for the $m=3$ mode for the $(m=3, m=5)$ coupling (see Table II) as its value should remain close to the $\gamma_{2}$ value for the $(m=1, m=3)$ coupling (i.e., $\gamma^{\operatorname{EELS}}(m=3) \approx-0.04$ and $\left.\gamma^{\text {Light }}(m=3) \approx-4 \times 10^{-3}\right)$.

In the application of the ECO model to the case of electron excitation, in keeping with relative differences in modal weighting factors for light and electron 
excitation, ${ }^{67}$ the polarizability $\alpha$ can be considered an effective polarizability $\alpha_{e f f}=\alpha_{e l} \alpha_{i n}$ where the intrinsic, unvarying polarizability $\alpha_{i n}$ due to the mode and material is modified by the particular responsivity to the electron beam excitation $\alpha_{e l}$.

As a result of these fits, the coefficient $C_{1}$ approaches and even assumes slightly positive values at energies just below the $m=3$ resonance. This effect is even more pronounced for $C_{1}$ in the $(m=3, m=5)$ system, where the lower energy mode coefficient $C_{1}$ assumes positive values at energies just below the $m=5$ resonance (Fig. 11(b), right panel). In contrast, for light scattering, the coefficient $C_{1}$ remains negative at the $m=5$ resonance energy (Fig. 11(a)), consistent with the asymmetry noted in the net dipole moment. In the light scattering case, there is destructive interference between $C_{1}$ and $C_{2}$ just below the resonance energy and constructive interference above the resonance energy. For electron excitation, there is a significant reduction in destructive interference (Fig. $10(\mathrm{~b})$ ) or even constructive interference (Fig. 11(a)) below the resonance energy. The significantly more symmetric peak shape recorded in EELS and CL is consistent with the ECO model fitting suggesting a change in the characteristic destructive interference signature just below resonance (Fig. 10(d)).

For electron excitation, the peak shape at the $m=3$ resonance is relatively symmetric, allowing the further application of the ECO model to the $m=3$ and $m=5$ resonances (Fig. 11(b)). The quality of the fit for modes $m=3$ and $m=5$ (Fig. 11) is reduced relative to Fig. 10 as the system is only partially described by the ECO model in this case ( $m=1$ not included in the fit), particularly in the case of light excitation where the $m=3$ resonance is more strongly affected by the $m=1$ mode. Fitting results are presented in Table II. For additional interacting modes, the system of equations cannot be written simply in terms of normal coordinates, precluding fitting with fixed resonance energies. The two oscillator ECO model, however, is retained here for its intuitive analysis and to assist in multi-parameter fitting. It provides a useful semi-quantitative interpretation of the difference between light scattering and EELS and helps to identify the underlying physical differences in the observed near field response characteristics of the nanorod to light and electron beam excitation.

According to these ECO modeling results, the spectral peaks in electron excitation of $m=5$ correspond to the in-phase summation of longitudinal Fabry-Pérot modes above and below resonance. That is, the spatial amplitude modulation should be well-described as a linear combination of the spatially-defined Fabry-Pérot modes as in other hybridized Fano-resonant single particles. ${ }^{12,55}$ As suggested by López-Tejeira et al., the Fabry-Pérot modes can be written as basis functions given by: ${ }^{41}$

$$
\varphi_{n}(x)=\sin (n k x),
$$

where $\varphi_{n}$ is the spatial distribution of the surface charge
TABLE II. Fitting parameters for ECO modelling of light scattering and EELS $(m=3, m=5)$. Relative units are indicated in terms of energy units $(\mathrm{eV})$ following Ref. 51.

\begin{tabular}{ccc}
\hline \hline Parameter & $\begin{array}{c}\text { Light Scattering } \\
(m=3, m=5)\end{array}$ & $\begin{array}{c}\text { EELS } \\
(m=3, m=5)\end{array}$ \\
\hline$E_{0}$ & 1.0 & 1.0 \\
$\omega_{1}[\mathrm{eV}]$ & 1.34 & 1.34 \\
$\omega_{2}[\mathrm{eV}]$ & 1.98 & 1.98 \\
$g\left[\mathrm{eV}^{2}\right]$ & 0.140 & 0.140 \\
$\gamma_{1}[\mathrm{eV}]$ & $-4.07 \times 10^{-3}$ & -0.0377 \\
$\gamma_{2}[\mathrm{eV}]$ & -0.0543 & -0.0581 \\
$\alpha_{1}\left[\mathrm{eV}^{-1}\right]$ & 0.0550 & 0.0859 \\
$\alpha_{2}\left[\mathrm{eV}^{-1}\right]$ & 0.00970 & 0.0423 \\
\hline \hline
\end{tabular}

(or real part of the normal componet of the electric field) as a function of position $x, k$ is the spatial frequency given as $k=\pi / L_{\text {eff }}$ where $L_{\text {eff }}$ is the effective length of the rod, and $n$ is an integer given as $n=m+1$. The interaction of two modes is then given as:

$$
\psi_{m}(x)=a_{n} \varphi_{n}+a_{n-2} \varphi_{n-2},
$$

where now $\psi_{m}$ is the hybridized mode corresponding to the observed mode $m$ and $a_{n}$ is an amplitude coefficient. Applying a similar approach as in Ref. 51, the coefficients $C_{1}$ and $C_{2}$ from ECO model fitting can be applied as the coefficients $a_{n}$ and $a_{n-2}$. Then the real part, in keeping with the consideration of the real part of the electric field as a measure of the surface charge, ${ }^{41,51}$ becomes:

$$
\psi_{m}(x)=\operatorname{Re}\left\{C_{1}\right\} \varphi_{n}+\operatorname{Re}\left\{C_{2}\right\} \varphi_{n-2} .
$$

The results of this analysis for the $m=3$ and $m=5$ modes is schematically represented in Fig. 11(c) (for $m=5, n=6, n-2=4)$. This approach adheres to the principles of surface plasmon mode hybridization schemes, ${ }^{68}$ in that there are two spectral features that are effectively "bonding" and "antibonding" modes. At the $m=3$ energy, $\operatorname{Re}\left\{C_{1}\right\}$ and $\operatorname{Re}\left\{C_{2}\right\}$ have opposite signs and the result is the lower energy or "bonding" state. At the $m=5$ energy, $\operatorname{Re}\left\{C_{1}\right\}$ and $\operatorname{Re}\left\{C_{2}\right\}$ have the same sign and the result is the higher energy or "antibonding" state. Figure 11(c) presents a comparison of the spatial amplitude at an energy just below the $m=5$ resonance, an energy typically characterized in Fano-resonant systems by destructive interference and $\operatorname{Re}\left\{C_{1}\right\}$ and $\operatorname{Re}\left\{C_{2}\right\}$ with opposite signs. In the electron excited case, however, the ECO model fitting results show $\operatorname{Re}\left\{C_{1}\right\}$ and $\operatorname{Re}\left\{C_{2}\right\}$ have the same sign (Fig. 11(b)). Moreover, a direct comparison of the spatial amplitude modulation determined from the ECO model applied to Fabry-Pérot basis functions (Fig. 11(d), red solid line) and the EELS line profile (Fig. 11(d), black squares) shows remarkable agreement between the spatial amplitude along the rod length. The rod tips are excluded in this analysis as the EELS signal is weak and the distance from the tip to the 
a)

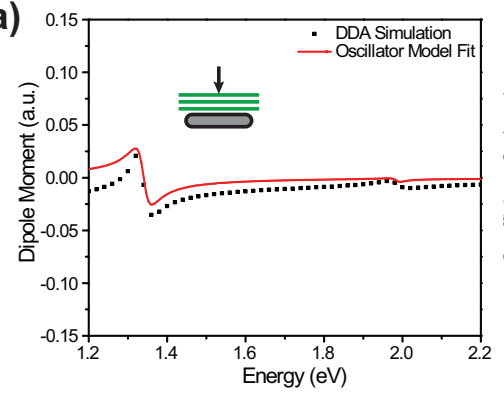

b)

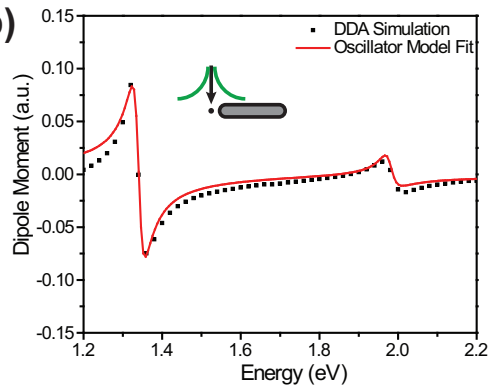

c)

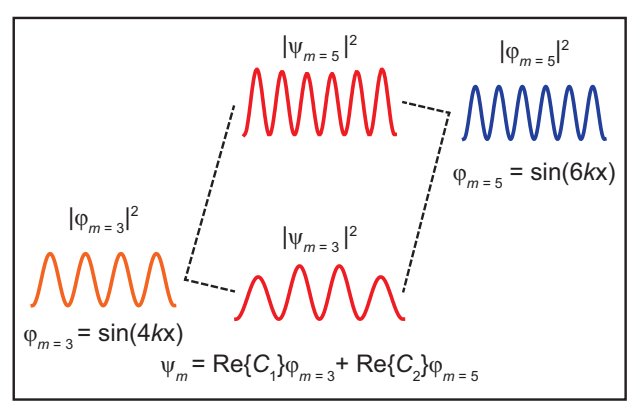

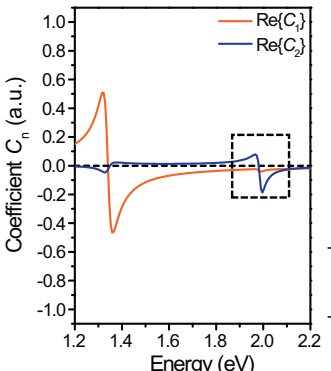
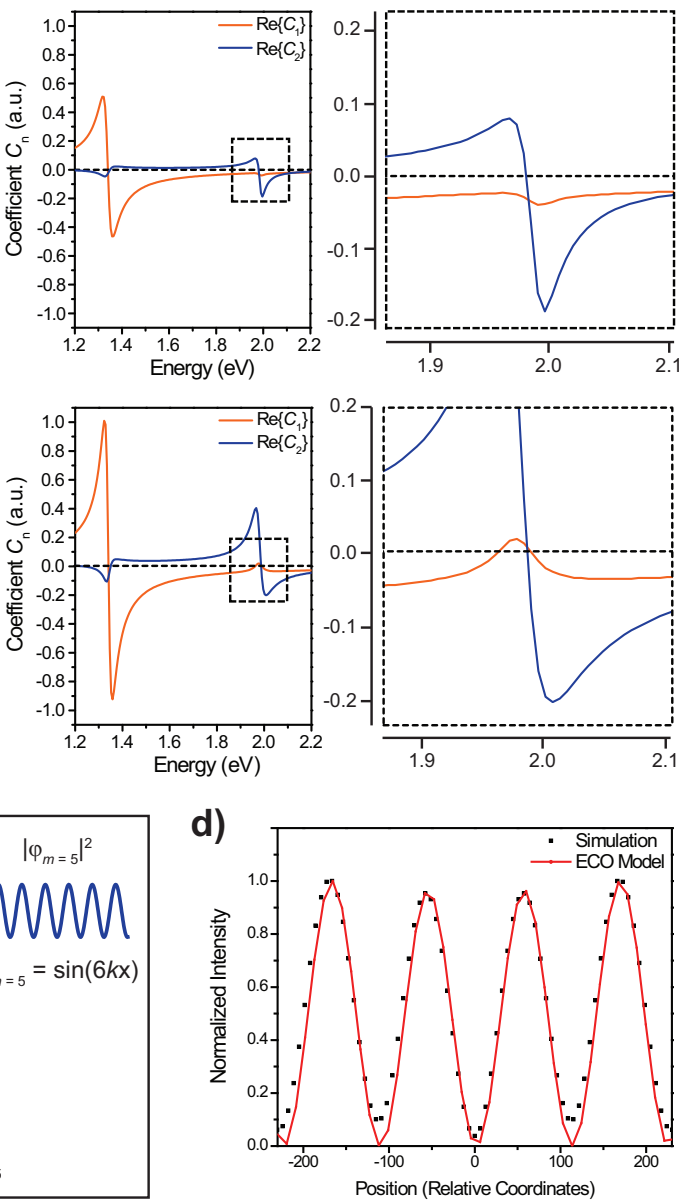

FIG. 11. (Color online) ECO model fitting of the dipole moment data from DDA simulations for (a) light scattering and (b) electron excitation of Rod A $(m=3, m=5)$. In the left panel, the dipole moment $P_{t o t}$ along the y-axis of the rod is plotted as well as best fit net dipole moment $P_{t o t}$ for the ECO model (Eq. 5). In the middle panel, the real coefficients for the two oscillators are plotted, and the signs of the coefficients are highlighted at the higher energy resonance in the right panel. (c) Hybridization diagram for interacting Fabry-Pérot basis modes $\varphi_{m}$ and the resulting hybridized mode $\psi_{m}$ at the $m=5$ resonance. (d) Comparison of the spatial amplitude modulation in the simulated EELS line profile and the spatial amplitude determined from a linear combination of Fabry-Pérot modes multiplied by the coefficients $C_{1}$ and $C_{2}$ extracted by ECO model fitting in (a).

next antinode is foreshortened. In the line profile, the electron trajectory is further from the rod surface due to the curvature of the rod tips (see also Sec. III-IV) and additionally the surface charges and associated fields curve three-dimensionally at the rod tips resulting in the observed shortening in the spacing between the antinodes at the tip when projecting onto a two-dimensional space as in the case of the EELS signal ( $x y$ space in Fig. 3(c)). The hybridization scheme in Fig. 11(c) for the "bonding" configuration also suggests the tips may be damped due to the hybridization at the $m=3$ resonance. However, this hybridization does not account for the contribution of the $m=1$ resonance on the $m=3$ spatial distribution which would enhance the intensity at the tips. Section VII examines a more flexible analysis to account for the interaction of more than two modes.

In this analysis, the spatial amplitude modulation ap- pears to be fully explained by the ECO modeling results. While other contributing effects cannot immediately be ruled out, the phase analysis from the ECO model suggests that for any approximately sinusoidal basis set, amplitude modulation will be introduced by the hybridization present in the electron excited rod and observed in the CL spectra (Fig. 7(d)) and dipole moments (Fig. 10-11) determined by DDA simulations. Notably, the ECO model fitting was performed for an electron trajectory at the rod tip, where all modes are excited. The spatial dependence of the EELS signal, however, is well described at least in the quasi-static approximation in terms of charges associated with the eigenmodes of the 
nanoparticle: $: 37,69$

$$
\begin{aligned}
& \Gamma\left(\mathbf{R}_{0}, \omega\right)=\frac{4}{\pi v^{2}} \sum_{i} \operatorname{Im}\left[-g_{i}(\omega)\right] \\
& \quad \times\left|\oint d \mathbf{s} \sigma^{i}(\mathbf{s}) e^{-i s^{\|}(\omega / v)} K_{0}\left(\frac{\omega\left|\mathbf{R}_{0}-s^{\perp}\right|}{v}\right)\right|^{2} .
\end{aligned}
$$

where here $\mathbf{R}_{0}$ is the position vector defining the electron trajectory, $\operatorname{Im}\left\{-g_{i}(\omega)\right\}$ gives a frequency dependent spectral intensity associated with the eigenmode $i, \sigma^{i}$ are the surface charges, and $\mathbf{s}$ defines the particle boundary with components parallel and perpendicular to the electron trajectory, and $K_{0}$ is the modified bessel function of the second kind.

In the framework of the modal decomposition, ${ }^{37}$ the unhybridized basis modes $\varphi_{n}$ should be considered as a description in a different basis than that of the eigenmodes. Boudarham and Kociak have reported quasistatic calculations for the electromagnetic local density of states (EMLDOS) of a rod exhibiting spatial amplitude modulation of the signal in rods with dissipative dielectric functions. ${ }^{37}$ The near fields associated with the eigencharges already exhibit spatial amplitude modulation, suggesting the eigenmodes may be the hybridized modes considered here. Recent analysis of Fano-resonances in silver cubes ${ }^{13}$ suggests that Fano-resonance phase analysis requires the appropriate basis, and in the case of these silver rods the Fabry-Pérot modes given in Eq. 7 appear to be suited for the phase analysis necessary to explain the line shapes of CL and light scattering and for the spatial amplitude modulation observed in CL and EELS. The eigenmode description suggests orthogonal modes, ${ }^{37}$ and Eq. 10 does not allow for coherent summation of eigenmodes as the sum is taken outside the squared modulus and given that $\operatorname{Im}\left[-g_{i}(\omega)\right]$ must be positive if each contribution to the sum gives a positive probability.

The silver nanorods examined here exhibit interference signatures in CL suggesting coherent summation of modes. Additionally, the spatial amplitude modulation signatures are present in experimental and simulated data before and after processing to separate the effects of spectral overlap (incoherent summation) by NMF decomposition of experimental results or peak fitting of simulated spectra. ${ }^{46}$ As such, the unhybridized modes do not appear to represent an orthogonal basis as enumerated in the quasi-static equations. The hybridized modes $\psi_{m}$ may provide such a basis. The differences identified between light and electron excitation suggest that the particular incident field plays a role in hybridization, and so retardation effects may also need to be included to fully explain the results of the DDA simulations and EELS experiments (see also Sec. VI). Critically, even if operating on a different basis, Eq. 10 establishes a qualitative connection between the surface charges and the expected spatially varying EELS signal. Eq. 10 gives this connection between complex surface charges and the EELS signal as the squared modulus of a surface integral over the charges and terms related to the exciting electron beam with the sum over eigenmodes taken outside the squared modulus. The appearance of hybridization effects in electron spectra (CL spectra in particular) invites further investigation to account for interference effects in analytical expressions describing the quasi-static or fully retarded cases.

Here, in keeping with the charge analyses in Ref. 41 and Ref. 51, $\psi_{m}$ is defined as in Eq. 9 and $\left|\psi_{m}\right|^{2}$ is plotted in Fig. 11(c)-(d). The phase of the interfering modes determined from ECO model fitting and their application to the Fabry-Pérot modes identified in Eq. 7 and Ref. 41 describe the observed spatial amplitude modulation in EELS simulations and experiments and indicate that spatial amplitude modulation is a signature of mode hybridization under electron excitation marked in the spectral domain by asymmetric line shapes in CL.

\section{TUNING NANOROD MODE HYBRIDIZATION}

In order to further elaborate the connection between the observed Fano-like resonance in the spectral domain and the spatial amplitude observed in EELS simulations and experiments, the tuning of these two observables was examined by exploring the effect of internal damping and the incident electron beam energy. These parameters alter the spectral overlap of the interacting modes and modify the incident field, respectively, and as such should (following the explanations offered in Sec. V) change the signatures of hybridization of longitudinal rod modes in the spectral and spatial domains. The line shape of individual peaks can be analyzed in terms of the Fano line shape: ${ }^{1}$

$$
I(E)=\frac{\left(q \Gamma+E-E_{r e s}\right)^{2}}{\left(E-E_{r e s}\right)^{2}+\Gamma^{2}},
$$

where $I$ is the signal intensity as a function of energy $E, \Gamma$ describes the width of the peak, $E_{r e s}$ is the resonance energy, and $q$ is the Fano parameter. The Fano or asymmetry parameter $q$ determines both the intensity and the asymmetry of the peak. ${ }^{1,70}$ As the parameter $q$ varies from 0 to 1 , the line shape evolves from a dip on resonance to an asymmetric peak, and as the parameter varies from 1 to $\infty$ the peak tends toward a symmetric Lorentzian line shape. ${ }^{70}$ Here, we modify the Fano line shape to account for any background in the CL signal with a simple linear background across the width of the peak:

$$
I^{\mathrm{CL}}(E)=\frac{\left(q^{\mathrm{CL}} \Gamma+E-E_{r e s}\right)^{2}}{\left(E-E_{r e s}\right)^{2}+\Gamma^{2}}+(a E+b),
$$

where now $I^{\mathrm{CL}}$ is the CL intensity as a function of energy $E$ and $a$ and $b$ give the slope and offset of a linear and incoherent background. The high quality of the fit of Eq. 12 to simulated CL spectra is depicted in the inset of Fig. $13(\mathrm{a})$. 
Figure 12 presents EELS and light scattering simulations for varied damping parameter $\Gamma$ for a Drude model dielectric function. This Drude model dielectric function matches the Johnson and Christy data ${ }^{53}$ for $\Gamma \approx 0.02 .{ }^{55}$ For the EELS signals (Fig. 12(a)), the spatial amplitude modulation increases with additional internal damping. For reduced internal damping, the spatial amplitude modulation remains unchanged. Simultaneously, the asymmetry parameter for the CL signal $q^{\mathrm{CL}}$ decreases from 3.94 to 1.43 with increased damping, corresponding to a significant increase in the peak asymmetry, and increases slightly to 4.98 for reduced damping. While it appears that the absence of a change in the spatial amplitude modulation for reduced damping may contrast with the change in the peak asymmetry, comparison with light scattering simulations (Fig. 12(b)-(c)) suggests that the spatial amplitude modulation in both light scattering and EELS may be at a limiting case for internal damping in the case of the particular simulated $\operatorname{rod}(\operatorname{Rod} \mathrm{A})$. In fact, light scattering simulations for $\Gamma=0$, not feasible for electron excitation due to slow numerical convergence, confirm that even with no internal damping, the far field scattering spectrum exhibits an asymmetric line shape (Fig. 12(b)). Moroever, the light scattered near field (Fig. 12(c)) shows imperceptible differences from $\Gamma=0$ to $\Gamma=0.02$. Increased damping $(\Gamma=0.20)$ results in noticeable changes to the near field in light scattering as for the EELS line profiles. As discussed in Sec. V, the phase relationships and relative mode polarization differ for light and electron excitation, but the qualitative comparison here emphasizes similarity in the damping dependence. In the case of Rod A, the damping dependence suggests that increasing the peak widths increases the coupling between the modes, resulting in more asymmetric line shapes in CL and light scattering. In the spatial domain, increased damping and increased coupling correspond to an increased modulation of the antinode intensity for EELS and light scattering. In the simulations here, the peak widths are due to intrinsic internal damping properties of the material and are not due to experimental broadending due to the finite width of the ZLP which contributes to incoherent blurring of the spectra only (see also $\mathrm{SM}^{46}$ ). It is clear from the line shape analysis that the interference effects approach a limiting case as a function of internal damping as the $\Gamma$ tends toward 0 ; the spatial amplitude modulation likewise reaches a limiting case though there is not a linear correspondence between the spectral and spatial effects. It should be noted that the scaling of the Fano parameter is also nonlinear, in that symmetric Lorentzian peaks are recovered only in the limit as $q$ tends to $\infty$ for Eq. 11. The increase in $q$ from 3.94 to 4.98 for smaller internal damping parameter $\Gamma$ reflects a relatively less significant change in peak asymmetry than the decrease to 1.43 for greater internal damping. Radiative damping is still present with no internal damping, and so likely explains the persistence of interference between the modes at $\Gamma=0$. Additional simulations on higher order modes

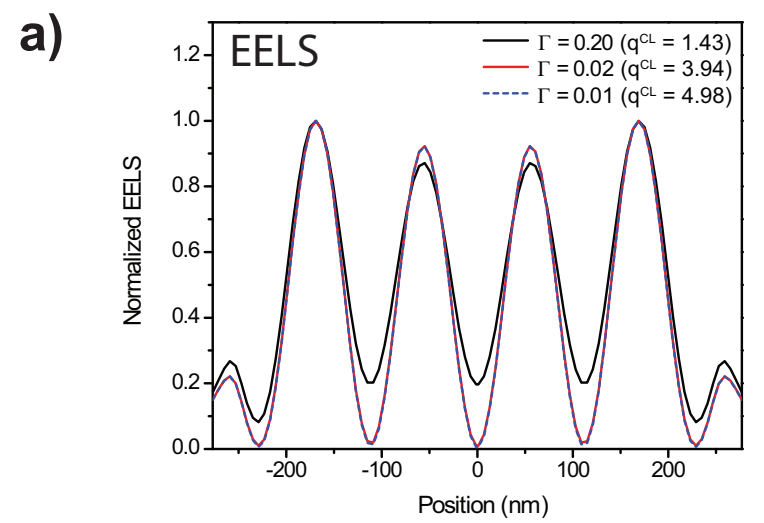

b)

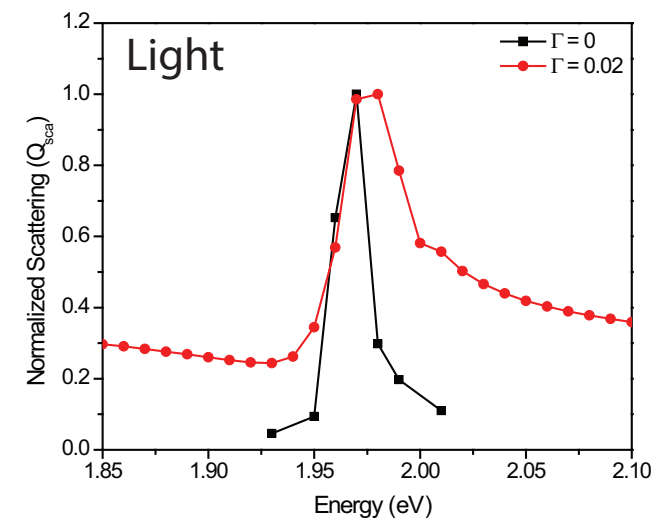

c)

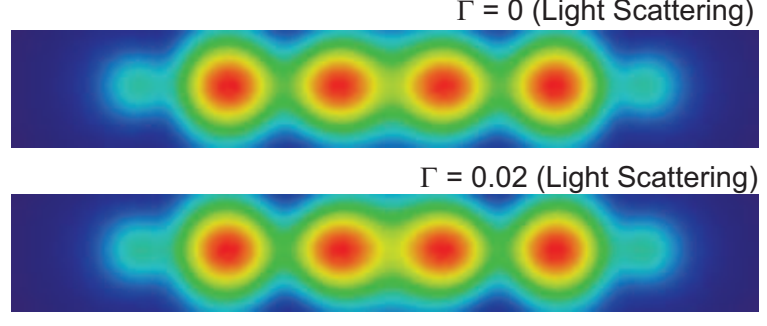

$\Gamma=0.20$ (Light Scattering)

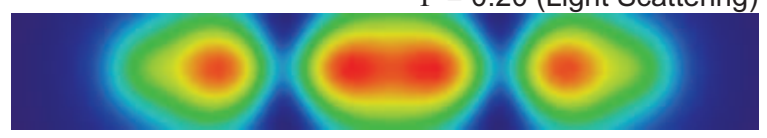

FIG. 12. (Color online) (a) EELS line profiles along Rod A (4 nm offset) with varying internal damping parameter $\Gamma$ calculated with a Drude model dielectric function. (b) Far field light scattering spectra for zero internal damping and a best fit Drude model dielectric function for silver. (c) Near fields at planes (c) $40 \mathrm{~nm}$ above the rod on resonance with the $m=5$ mode at $1.97 \mathrm{eV}$ for these simulations.

with reduced radiative damping exhibit less spatial amplitude modulation. ${ }^{46}$

In principle, removing damping effects altogether might present a test case to examine these near field interference effects in silver rods. However, in order to remove damping entirely, the peak widths would simultaneously tend toward zero, limiting the feasibility for numerical calculations scanning the energy spectrum. The asymmetry in the CL and light scattering peaks even at very low damping suggests that interference effects are 
still present for narrow and spectrally resolved peaks in this system. The ECO model fitting (Sec. V) highlights that coupled oscillators with resonances separated significantly in energy can still act on each other suggesting spectral line width may not be a simple predictor of mode interaction.

The damping dependence demonstrates that the spatial amplitude modulation and spectral asymmetry for electron and light excitation is not purely a geometric effect due to the shape of the rod. The damping dependence, however, does not on its own rule out dissipation in the rod as an independent source of the spatial amplitude modulation. Consequently, separate simulations were carried out to tune the hybridization of the rod modes by altering the exciting field only. With electron excitation, the incident field can be tuned readily by adjusting the incident beam energy. In the frequency domain, the electric field components of the incident electron are given in terms of modified bessel functions with arguments inversely proportional to the electron velocity $\left(1 / v_{e l}\right) .{ }^{59}$ At lower incident electron energies and lower incident electron velocities, the arguments of the modified bessel functions increase and consequently the fields exhibit a more localized spatial distribution at lower electron energies. Figure 13(a) presents a series of CL simulations for Rod A (no substrate) for $80 \mathrm{keV}, 200 \mathrm{keV}$, and $300 \mathrm{keV}$, energies corresponding to typical accelerating voltages in STEM-EELS experiments. The intensity of modes $m=4-6$ increases as the incident electron energy is reduced. These results are consistent with the energy dependence of higher order modes in spheres $;^{71}$ the lower energy electron couples more efficiently to higher order modes because its field is more local and therefore matches the higher spatial frequencies required to couple to multipolar modes. Figure 13(b) shows the associated values of $q^{\mathrm{CL}}$ determined by fitting the spectra in Fig. 13(a) to Eq. 12. The concomitant changes in incident electron energy and the Fano parameter $q^{\mathrm{CL}}$ highlight that when the relative polarization of modes in a coupled system changes so too does the observed line shape. In the ECO model (Sec. V), greater polarization of the higher energy mode for electron excitation relative to plane wave light corresponds to reduced asymmetry in the scattering spectrum (Fig. 10(a)-(b), right panels). In Fig. 13, as the electron energy is reduced, these higher order modes are excited more intensely and the spectral line shapes become more symmetric ( $q^{\mathrm{CL}}$ is higher for lower incident electron energies). This tunability of the CL line shape with electron energy underscores the excitation dependence of the Fano-like resonance signature in these rods.

To correlate the effect of the incident electron energy on the spatial amplitude modulation observed in EELS, a simple phenomenological measure is given by the contrast $C^{\text {EELS: }}$

$$
C^{\mathrm{EELS}}=\frac{I_{\max }-I_{\min }}{I_{\max }+I_{\min }}
$$

where $I_{\max }$ refers to the simulated EELS signal at the
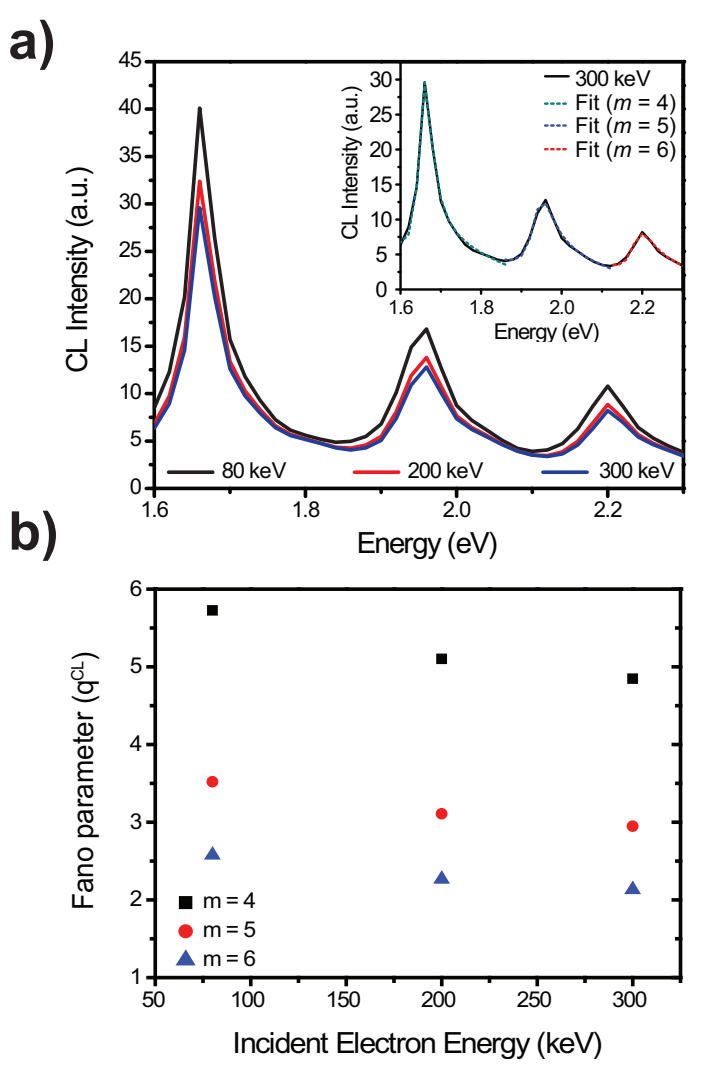

FIG. 13. (Color online) (a) CL spectra for Rod A (no substrate) for $80 \mathrm{keV}, 200 \mathrm{keV}$, and $300 \mathrm{keV}$ incident electron beam energies. The inset shows example fitting of each peak ( $m=4-6)$ using Eq. 12 for the $300 \mathrm{kV}$ CL spectrum. (b) The Fano parameter $q^{\mathrm{CL}}$ determined by fitting Eq. 12 to the spectra in (a) for modes $m=4-6$.

antinode (the peak in the line profile) with the greatest intensity and $I_{\text {min }}$ refers to the simulated EELS signal at the antinode with the lowest intensity, excluding the tips in the line profile where the signal is low for all modes.

Table III presents this antinode contrast and compares the effect of incident electron energy on $q^{\mathrm{CL}}$ and on $C^{\mathrm{EELS}}$. The correlation between electron energy and antinode contrast is consistent for each of the three modes, with antinode contrast increasing as the Fano parameter $q^{\mathrm{CL}}$ decreases and the line shape asymmetry increases from $80 \mathrm{keV}$ to $300 \mathrm{keV}$ incident electron energy. The particular values of $C^{\text {EELS }}$ are not directly comparable between the different modes as they describe antinodes at different positions along the rod. In this system, the changes in line shape asymmetry directly describes a modification of the interaction of interfering modes, and the reduced antinode contrast at lower incident electron energies corresponds to increasingly equal intensity at all antinodes along the rod. Moreover, this consistent decrease in antinode contrast at lower incident electron energies for each mode matches experimental observations carried out on longer rods at $80 \mathrm{keV}$ where minimal spatial amplitude modulation was observed. ${ }^{25}$ The CL sim- 
TABLE III. Comparison of the CL Fano parameter $q^{\mathrm{CL}}$ and EELS antinode contrast $C^{\text {EELS }}$ for incident electron energies of $80 \mathrm{keV}, 200 \mathrm{keV}$, and $300 \mathrm{keV}$ and modes $m=4-6(\operatorname{Rod}$ A).

\begin{tabular}{|c|c|c|c|}
\hline Mode & Electron Energy (keV) & $q^{\mathrm{CL}}$ & $C^{\mathrm{EELS}}$ \\
\hline \multirow{3}{*}{$m=4$} & 80 & 5.72 & 0.0058 \\
\hline & 200 & 5.10 & 0.059 \\
\hline & 300 & 4.85 & 0.10 \\
\hline \multirow{3}{*}{$m=5$} & 80 & 3.52 & -0.0032 \\
\hline & 200 & 3.11 & 0.033 \\
\hline & 300 & 2.95 & 0.051 \\
\hline \multirow{3}{*}{$m=6$} & 80 & 2.58 & 0.020 \\
\hline & 200 & 2.27 & 0.071 \\
\hline & 300 & 2.14 & 0.11 \\
\hline
\end{tabular}

ulations function as a tool for assessing the changes to the Fano-like interference effects as a result of mode hybridization in these silver rods, and the parameter $q^{\mathrm{CL}}$ appears to be a consistent predictor of changes in the simulated EELS antinode contrast $C^{\text {EELS }}$.

Taken together, the tunability of the line shape asymmetry and concomitant changes in spatial amplidude modulation present a strong case for identifying the spatial amplitude modulation as a signature of the Fano-like interference effect in the longitudinal modes of these silver nanorods. Damping properties of the rod are not modified when changing the electron energy, and so internal damping of the surface plasmon does not explain the increasingly equal spatial intensity Fabry-Pérot patterns observed at lower electron energies. In fact, the contrast even changes sign (i.e., the antinode positions corresponding to $I_{\max }$ and $I_{\min }$ are reversed) for $m=5$ at 80 $\mathrm{keV}$ (Table III). Such a change in contrast is consistent with the hybridization model, explained as a change of the relative weighting factors of the contributing modes such as an increasing role of $m=7$ and higher order modes in the hybridization scheme. Geometric properties of the rod or material properties (damping) do not explain both the damping and the electron energy dependencies observed for the spatial amplitude modulation, whereas the Fano parameter and associated trends in line shape asymmetry explain the associated changes in spatial amplitude modulation for the nanorods examined here.

The length of the rod provides yet a further tunable parameter, and for longer rods, size effects lead to greater spectral separation of the modes as the modes red-shift to lower energies. For the same mode $m$, the interacting modes are separated more in energy for longer rods, and this greater energy separation should reduce the magnitude of the interference effect. Spatial amplitude modulation does in fact decrease for the same mode $m$ (see $\mathrm{SM}^{46}$ ), consistent with the expected reduction in coupling between the interfering modes for increased rod length. This section is limited in scope to a perturbative examination of the effects of changes to material properties and the exciting field on simulated CL line shape asymmetries and EELS spatial amplitude modulation for the silver rods examined in Sec. III-V. More extensive studies of different rod lengths, materials, and incident electron energies both through simulation and experimental observation alongside further experimental work investigating the CL line shape and correlated light and electron studies will be required to fully describe the Fano-like interference effects in nanorods more generally. Cumulatively, considering the spatial amplitude modulation determined from the ECO model (Sec. V, Fig. 11) as well as the consistent trends in tuning the damping and electron beam excitation, the spatial amplitude modulation in EELS represents a signature of the Fano-like interference in the silver nanorods examined here. Other effects, as yet not explained, might contribute as well, but the interference effects observed for electron beam excitation by CL describe a near field spatial interference pattern reproduced in the spatial amplitude modulation of the EELS signal.

\section{SPATIAL AMPLITUDE MODULATION FITTING}

In previous examinations of near field interference in light scattering of nanorods, relative contributions of the various modes were estimated by fitting fields near the rod, a proxy variable for surface charge density, to a sum of sinusoidal basis functions ${ }^{41}$ (see also Sec. V). The ECO model approach in Sec. V, however, is largely limited to examining the interaction of two modes. In electron excitation, the higher order multipolar resonances are excited more strongly than in light scattering (see aslo Fig. 10-11) and there is no general reason to believe that only a single mode contributes to the interference effects observed in light scattering and in electron excited spectra. In light scattering, the higher order modes are excited weakly and the Fano-like resonance appears to be well described by interaction with the next lower energy odd mode only. ${ }^{36,41}$ To explore possible contributions of other modes beyond the ECO model, Eq. 10 can be generalized as a sum over additional modes and fit to EELS line profiles. Such an approach, while not as analytical as the decomposition offered by the ECO model, is more flexible and offers a means to understand the relative contributions of the interfering Fabry-Pérot modes of the silver nanorods examined here.

In a similar approach to Ref. 41 and extending Eq. 10 as a sum over several modes gives:

$$
\Gamma(x)=\left[\sum_{n}^{m+1} a_{n} \sin (n k(x-b))\right]^{2}+y_{0},
$$

where the spatial frequency is likewise $k=\pi / L_{\text {eff }}$ where $L_{e f f}$ is the effective length of the rod, $x$ is the position 
TABLE IV. Mode coefficients $a_{n}$ (Eq. 14) corresponding to simulated line profiles plotted in Fig. 14 as well as results for Rod B (not shown). Relative contributions have been normalized to sum to unity.

\begin{tabular}{llccc}
\hline \hline & & $n_{m}$ & $n_{m}-2$ & $n_{m}-4$ \\
\hline \multirow{4}{*}{$\operatorname{Rod}$ A } & $m=4$ & 0.955 & 0.045 & - \\
& $m=5$ & 0.965 & 0.035 & $\sim 0$ \\
& $m=6$ & 0.870 & 0.081 & 0.049 \\
\hline \multirow{3}{*}{$\operatorname{Rod}$ B } & $m=4$ & 0.947 & 0.053 & - \\
& $m=5$ & 0.950 & 0.050 & $\sim 0$ \\
& $m=6$ & 0.821 & 0.122 & 0.051 \\
\hline \hline
\end{tabular}

along the rod, $b$ is an offset to center the sinusoid at the middle of the rod $(x=0), y_{0}$ is an offset on the intensity axis due to any background, and $n$ is incremented such that only corresponding odd or even sinusoids from the two preceding modes are included in the summation (e.g., for $m=6, n=\{3,5,7\}$, and $n_{m}=m+1=7$ ). Only two lower order resonances of the same symmetry are considered to avoid overfitting (in total, no more than three sinusoids in Eq. 14); lower energy resonances are more highly excited and thus contribute predominantly to the observed amplitude modulation. Additional higher order modes could be included, but these were not required in a minimal fitting procedure for modes $m=4-6$ for Rod $\mathrm{A}$ and Rod B.

The results of fitting Eq. 14 to the simulated line profiles are shown in Fig. 14. The rod tips are excluded from this fitting procedure as the signal at the tips is low due to various geometric factors ("end effects") discussed in Sec. III-IV. The remarkably good fit further corroborates the mode hybridization suggested by the ECO model. Whilst Eq. 14 cannot capture in a quantitative way the interaction of the Fabry-Pérot modes, the dependence of EELS on surface charges enables the fit using Eq. 14 to offer a signal proportional to the relative contributions of those modes; these are shown in Table IV. Fitting results show consistent modal contributions for Rod A and Rod B, with Rod B exhibiting higher coefficients for the lower energy $n_{m}-2$ mode, consistent with its shorter length. As a shorter rod in a lower refractive index environment, the Rod B modes are more closely spaced in energy and are expected to be more strongly coupled. The fitting results match this expectation in the simulated rods. Moreover, the relative contributions are of the order of a few percent, consistent with the relative magnitudes of $C_{1}$ and $C_{2}$ in the dipole moment fitting results (Table I, Fig. 11).

Notably, for the $m=6$ mode, both the $m=2$ and $m=4$ modes are necessarily included to reproduce the amplitude modulation (see also Fig. 4, Fig. 15). ${ }^{46}$ As all of these modes have the same symmetry, multiple mode orders can interact. Modes of different symmetry do not interact with each other in light scattering studies even when "dark" modes are excited with inclined
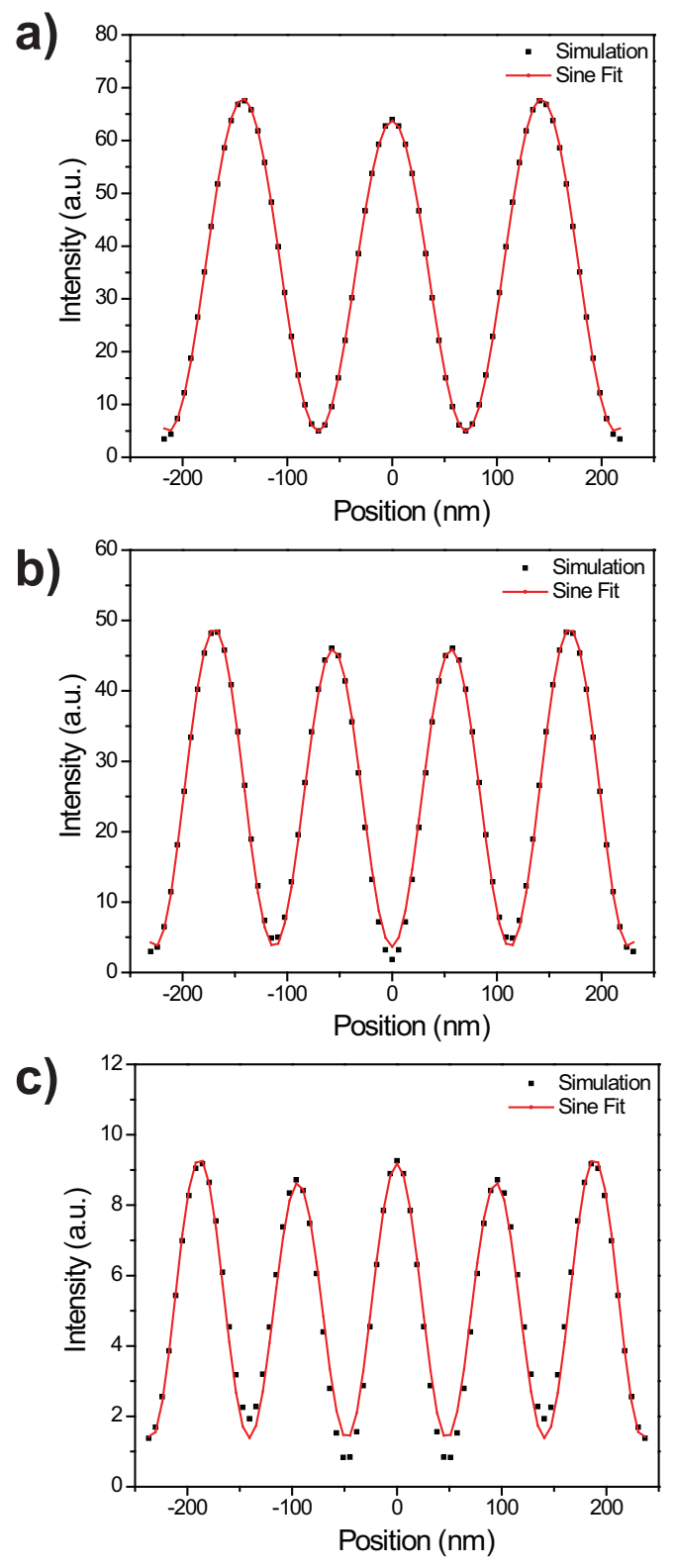

FIG. 14. (Color online) Simulated line profiles for Rod A (black squares) and results of fitting Eq. 14 to the simulated profiles (red lines). Eq. 14, a sum of sine functions, describes the relative contributions of interfering modes present in the EELS signal (excluding the rod tips) for (a) $m=4$, (b) $m=5$, and (c) $m=6$.

incident plane waves. ${ }^{36,41}$ The observed amplitude modulation in EELS is likewise consistent with only modes of the same symmetry interacting. The majority of analyses presented here have specifically involved "bright" modes as they more readily lend themselves to dipole moment analysis, but the nature of the interaction for electrondriven resonances is not fundamentally different as both "bright" and "dark" modes give rise to asymmetric CL 
TABLE V. Mode coefficients $a_{n}$ (Eq. 14) corresponding to experimental line profiles plotted in Fig. 15. Relative contributions have been normalized to sum to unity.

\begin{tabular}{llccc}
\hline \hline & & $n_{m}$ & $n_{m}-2$ & $n_{m}-4$ \\
\hline \multirow{2}{*}{ Rod A } & $m=4$ & 0.758 & 0.242 & - \\
& $m=5$ & 0.848 & 0.152 & $\sim 0$ \\
\hline \multirow{3}{*}{$\operatorname{Rod}$ B } & $m=4$ & 0.851 & 0.149 & - \\
& $m=5$ & 0.850 & 0.150 & $\sim 0$ \\
& $m=6$ & 0.600 & 0.234 & 0.167 \\
\hline \hline
\end{tabular}

line shapes (Fig. 7(d), Fig. 14(a)) and amplitude modulation is observed for both symmetries. The inclusion of mode $m=2$ in fitting Eq. 14 to the $m=6$ resonance is also justified in considering the role of any background continuum as proposed for light scattering analyses. ${ }^{36}$ The illuminating field of a swift electron exhibits mirror plane symmetry ${ }^{59}$ and consequently a continuum background excitation in the rod will exhibit a charge distribution similar to the $m=2$ mode in order to maintain charge neutrality in the rod.

Experimental line profiles are likewise suited to fitting to Eq. 14, and similar trends in modal contributions are observed. Results for fitting Eq. 14 to experimental line profiles are presented in Fig. 15. However, additional experimental factors including damping due to oxidation, carbon contamination during electron beam exposure, substrate effects, signal noise, and the inhomogeneous local dielectric environment for Rod $\mathrm{B}$ alter the relative contributions of the modes (Table V). The fitting results for simulated line profiles (Fig. 14) are presented as a self-consistent set with ECO model fitting results presented in Fig. 10 and Fig. 11. The spatial modulation fitting presented in this section serves to highlight features consistent with the interaction of more than two Fabry-Pérot modes in silver nanorods and to provide a simple tool for the assessment of the contributing modes in experimental data first presented in Sec. III.

\section{SUMMARY}

The plasmonic modes of silver nanorods with multipolar excitations at visible light energies were examined by analyzing experimental EELS maps using a decomposition approach (NMF) to separate the spectral and spatial features. These silver nanorods exhibited modes characteristic of Fabry-Pérot-like standing waves along the long axis of the rod, though significant modulation of the EELS signal at antinodes in the standing wave pattern were noted. Line profiles were extracted from experimental maps and were found to be in good agreement with DDA simulations.

To understand the observed spatial amplitude modulation in the experimental and simulated EELS line pro-
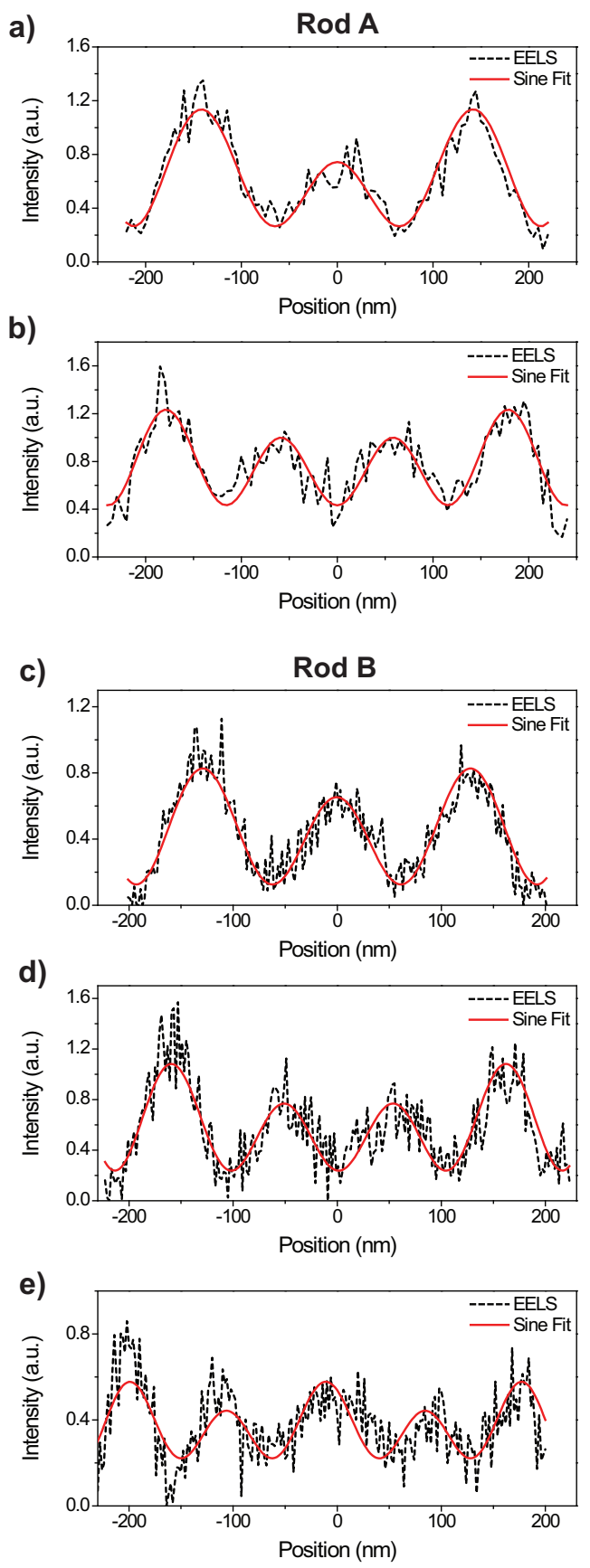

FIG. 15. (Color online) Experimental line profiles (dashed black line) and results of fitting Eq. 14 to the experimental line profiles (solid red lines) for $\operatorname{Rod} \mathrm{A}$ (a) $m=4$, (b) $m=5$ and $\operatorname{Rod} \mathrm{B}$ (c) $m=4$, (d) $m=5$, (e) $m=6$. Eq. 14, a sum of sine functions, describes the relative contributions of interfering modes present in the EELS signal (excluding the rod tips).

files, DDA simulations for light scattering, EELS, and CL were carried out. Light scattering simulations exhibited a Fano-like resonance in the spectral domain, consistent with previous reports on plasmonic nanorods. ${ }^{36,41}$ 
For the electron excited spectra, CL likewise exhibited a Fano-like asymmetry confirming interference effects for electron excitation. The CL spectra, however, exhibit distinct spectral asymmetry relative to light scattering spectra, particularly in the observation of asymmetric spectral peaks for both odd and even modes. ECO model fitting of the energy-dependent dipole moment for light and electron excitation revealed differences in the hybridization of the longitudinal rod modes for light and electron excitation. Application of the modal phase analysis by the ECO model to a simple Fabry-Pérot model for the spatial distribution of the longitudinal modes reproduced the observed spatial amplitude modulation in EELS and CL signals.

Additional examination of the spatial amplitude modulation and the CL peak asymmetry for internal damping and electron beam energy demonstrated systematic trends in changes to the interference signature in the CL spectra and the spatial amplitude modulation, attributed to changes to the relative coupling and polarization of the interfering longitudinal modes. A simple fitting procedure was reported to further probe the interaction of multiple resonances and was applied to qualitatively assess the relative modal contributions in experimental EELS line profiles.

The demonstrated difference in recorded interference in EELS and light scattering serves as motivation to study differences in other Fano-resonant plasmonic nanoparticle systems for electron and light spectroscopies. The particular amplitude modulation patterns analyzed here are potentially representative of a class of quasi-one dimensional multipolar modes, and related systems such as modes in kinked wires, ${ }^{25}$ asymmetric rods, ${ }^{72}$ and higher order modes of nanoparticle and nanohole edges ${ }^{73,74}$ are suitable candidates for similar hybridization effects. This work also informs further development and evaluation of SPR sensors and devices derived from metal nanorods using other excitations such as shaped near field excitation ${ }^{75}$ or unconven- tional polarization. ${ }^{76}$ Efforts to couple quantum $\operatorname{dots}{ }^{77,78}$ or absorptive layers ${ }^{79}$ in the near field of nanorods will benefit from consideration of local field enhancement at antinodes as a function of location on the nanorod. Understanding these single particle interference effects enables improved electron beam characterization of plasmonic near fields and makes available conceptual building blocks for more sophisticated plasmonic systems and devices.

\section{ACKNOWLEDGMENTS}

S.M.C. acknowledges support of a Gates Cambridge Scholarship. D.R. acknowledges support from the Royal Society's Newton International Fellowship scheme. We acknowledge the use of computing facilities provided by CamGrid. Parts of this work were also performed using the Darwin Supercomputer of the University of Cambridge High Performance Computing Service (http://www.hpc.cam.ac.uk/), provided by Dell Inc. using Strategic Research Infrastructure Funding from the Higher Education Funding Council for England and funding from the Science and Technology Facilities Council. We thank F.J. de la Peña for helpful discussions on the use of HYPERSPY. The research leading to these results has received funding from the European Research Council under the European Union's Seventh Framework Program (No. FP7/2007-2013)/ERC Grant Agreement No. 291522-3DIMAGE. Data on rod "B" was acquired by one of us (D. Rossouw) with support of a NSERC Discovery Grant (G.A. Botton) at the Canadian Centre for Electron Microscopy, a national facility supported by NSERC and McMaster University. We thank G.A. Botton for access to data on rod "B" and for helpful comments on this manuscript. P.A.M. also acknowledges funding from the European Union's Seventh Framework Program under a contract for an Integrated Infrastructure Initiative (Reference No. 312483-ESTEEM2).
* smc204@cam.ac.uk

† pam33@cam.ac.uk; www-hrem.msm.cam.ac.uk

1 B. Luk'yanchuk, N. I. Zheludev, S. A. Maier, N. J. Halas, P. Nordlander, H. Giessen, and C. T. Chong, Nat. Mater. 9, 707 (2010).

2 N. Verellen, P. Van Dorpe, C. Huang, K. Lodewijks, G. A. E. Vandenbosch, L. Lagae, and V. V. Moshchalkov, Nano Lett. 11, 391 (2011).

3 N. Liu, T. Weiss, M. Mesch, L. Langguth, U. Eigenthaler, M. Hirscher, C. Sönnichsen, and H. Giessen, Nano Lett. 10, 1103 (2010).

4 B. Lahiri, A. Z. Khokhar, R. M. De La Rue, S. G. McMeekin, and N. P. Johnson, Opt. Express 17, 1107 (2009).

5 N. Liu, M. Hentschel, T. Weiss, A. P. Alivisatos, and H. Giessen, Science 332, 1407 (2011), PMID: 21680838.

6 B. Gallinet, T. Siegfried, H. Sigg, P. Nordlander, and
O. J. F. Martin, Nano Lett. 13, 497 (2013).

7 W.-S. Chang, J. B. Lassiter, P. Swanglap, H. Sobhani, S. Khatua, P. Nordlander, N. J. Halas, and S. Link, Nano Lett. 12, 4977 (2012).

8 J. Wang, B. Yuan, C. Fan, J. He, P. Ding, Q. Xue, and E. Liang, Optics Express 21, 25159 (2013).

9 S.-O. Guillaume, N. Geuquet, and L. Henrard, Proc. SPIE 8096, 80962E (2011).

10 J. B. Lassiter, H. Sobhani, M. W. Knight, W. S. Mielczarek, P. Nordlander, and N. J. Halas, Nano Lett. 12, 1058 (2012).

11 N. W. Bigelow, A. Vaschillo, J. P. Camden, and D. J. Masiello, ACS Nano 7, 4511 (2013).

12 O. Nicoletti, F. de la Peña, R. K. Leary, D. J. Holland, C. Ducati, and P. A. Midgley, Nature 502, 80 (2013).

13 V. Iberi, N. W. Bigelow, N. Mirsaleh-Kohan, S. Griffin, P. D. Simmons, B. S. Guiton, D. J. Masiello, and J. P. 
Camden, J. Phys. Chem. C 118, 10254 (2014).

14 T. Ming, L. Zhao, Z. Yang, H. Chen, L. Sun, J. Wang, and C. Yan, Nano Lett. 9, 3896 (2009).

15 G. W. Bryant, F. J. García de Abajo, and J. Aizpurua, Nano Lett. 8, 631 (2008).

16 J. Aizpurua, G. W. Bryant, L. J. Richter, F. J. García de Abajo, B. K. Kelley, and T. Mallouk, Phys. Rev. B 71, 235420 (2005).

17 M. Bosman, V. J. Keast, M. Watanabe, A. I. Maaroof, and M. B. Cortie, Nanotechnology 18, 165505 (2007).

18 B. Schaffer, U. Hohenester, A. Trügler, and F. Hofer, Phys. Rev. B 79, 041401 (2009).

19 M.-W. Chu, V. Myroshnychenko, C. H. Chen, J.-P. Deng, C.-Y. Mou, and F. J. García de Abajo, Nano Lett. 9, 399 (2009).

20 M. N'Gom, S. Li, G. Schatz, R. Erni, A. Agarwal, N. Kotov, and T. B. Norris, Phys. Rev. B 80, 113411 (2009).

21 O. Nicoletti, M. Wubs, N. A. Mortensen, W. Sigle, P. A. van Aken, and P. A. Midgley, Opt. Express 19, 15371 (2011).

22 D. Rossouw, M. Couillard, J. Vickery, E. Kumacheva, and G. A. Botton, Nano Lett. 11, 1499 (2011).

23 B. Rodríguez-González, F. Attouchi, M. F. Cardinal, V. Myroshnychenko, O. Stéphan, F. J. García de Abajo, L. M. Liz-Marzán, and M. Kociak, Langmuir 28, 9063 (2012).

24 M. Bosman, E. Ye, S. F. Tan, C. A. Nijhuis, J. K. W. Yang, R. Marty, A. Mlayah, A. Arbouet, C. Girard, and M.-Y. Han, Sci. Rep. 3, 1312 (2013).

25 D. Rossouw and G. A. Botton, Phys. Rev. Lett. 110, 066801 (2013).

26 R. Gómez-Medina, N. Yamamoto, M. Nakano, and F. J. García de Abajo, New Journal of Physics 10, 105009 (2008).

27 T. Coenen, E. J. R. Vesseur, and A. Polman, ACS Nano 6, 1742 (2012).

28 J.-C. Weeber, A. Dereux, C. Girard, J. R. Krenn, and J.-P. Goudonnet, Phys. Rev. B 60, 9061 (1999).

${ }^{29}$ K. Imura, T. Nagahara, and H. Okamoto, The Journal of Chemical Physics 122, 154701 (2005).

30 J. Dorfmüller, R. Vogelgesang, R. T. Weitz, C. Rockstuhl, C. Etrich, T. Pertsch, F. Lederer, and K. Kern, Nano Lett. 9, 2372 (2009).

31 A. C. Jones, R. L. Olmon, S. E. Skrabalak, B. J. Wiley, Y. N. Xia, and M. B. Raschke, Nano Lett. 9, 2553 (2009).

32 J. Dorfmüller, R. Vogelgesang, W. Khunsin, C. Rockstuhl, C. Etrich, and K. Kern, Nano Lett. 10, 3596 (2010).

${ }^{33}$ L. Douillard, F. Charra, Z. Korczak, R. Bachelot, S. Kostcheev, G. Lerondel, P.-M. Adam, and P. Royer, Nano Lett. 8, 935 (2008).

34 E. S. Barnard, T. Coenen, E. J. R. Vesseur, A. Polman, and M. L. Brongersma, Nano Lett. 11, 4265 (2011).

35 E. S. Barnard, J. S. White, A. Chandran, and M. L. Brongersma, Optics Express 16, 1652916537 (2008).

${ }^{36}$ N. Verellen, F. López-Tejeira, R. Paniagua-Domínguez, D. Vercruysse, D. Denkova, L. Lagae, P. Van Dorpe, V. V. Moshchalkov, and J. A. Sánchez-Gil, Nano Lett. 14, 2322 (2014).

37 G. Boudarham and M. Kociak, Phys. Rev. B 85, 245447 (2012).

38 B. S. Guiton, V. Iberi, S. Li, D. N. Leonard, C. M. Parish, P. G. Kotula, M. Varela, G. C. Schatz, S. J. Pennycook, and J. P. Camden, Nano Lett. 11, 3482 (2011).

39 E. J. R. Vesseur, R. de Waele, M. Kuttge, and A. Polman,
Nano Lett. 7, 2843 (2007).

40 S. Zhang, L. Chen, Y. Huang, and H. Xu, Nanoscale 5, 6985 (2013).

41 F. López-Tejeira, R. Paniagua-Domínguez, R. RodríguezOliveros, and J. A. Sánchez-Gil, New J. Phys. 14, 023035 (2012).

42 K. E. Korte, S. E. Skrabalak, and Y. Xia, J. Mater. Chem. 18, 437 (2008).

43 F. de la Peña, M.-H. Berger, J.-F. Hochepied, F. Dynys, O. Stephan, and M. Walls, Ultramicroscopy 111, 169 (2011).

44 M. R. Keenan and P. G. Kotula, Surface and Interface Analysis 36, 203212 (2004).

45 C.-J. Lin, Neural Computation 19, 2756 (2007).

46 See Supplemental Material at [URL will be inserted by publisher] for further details of NMF processing, a discussion of the effects of the substrate and instrumental broadening, an evaluation of dipole discretization, additional internal damping calculations, additional spectral amplitude modulation fitting, additional simulations examining the role of rod length, and additional comments on related systems.

47 N. W. Bigelow, A. Vaschillo, V. Iberi, J. P. Camden, and D. J. Masiello, ACS Nano 6, 7497 (2012).

${ }^{48}$ P. J. Flatau and B. T. Draine, Journal of the Optical Society of America A 11, 1491 (1994).

49 P. J. Flatau and B. T. Draine, Optics Express 20, 1247 (2012).

50 N. Geuquet and L. Henrard, Ultramicroscopy 110, 1075 (2010).

51 A. Lovera, B. Gallinet, P. Nordlander, and O. J. Martin, ACS Nano 7, 4527 (2013).

${ }^{52}$ F. Zhou, Z.-Y. Li, Y. Liu, and Y. Xia, The Journal of Physical Chemistry C 112, 20233 (2008).

53 P. B. Johnson and R. W. Christy, Phys. Rev. B 6, 4370 (1972).

54 W. L. Johnson, S. A. Kim, Z. N. Utegulov, J. M. Shaw, and B. T. Draine, J. Phys. Chem. C 113, 14651 (2009).

55 S. Zhang, K. Bao, N. J. Halas, H. Xu, and P. Nordlander, Nano Lett. 11, 1657 (2011).

56 R. F. Egerton, Electron Energy-Loss Spectroscopy in the Electron Microscope, 3rd ed. (Springer, New York, 2011).

57 J. A. Aguiar, B. W. Reed, Q. M. Ramasse, R. Erni, and N. D. Browning, Ultramicroscopy 124, 130 (2013).

58 M. Stöger-Pollach, Micron 39, 1092 (2008).

59 F. J. García de Abajo, Rev. Mod. Phys. 82, 209 (2010).

60 J. Nelayah, M. Kociak, O. Stéphan, F. J. García de Abajo, M. Tencé, L. Henrard, D. Taverna, I. Pastoriza-Santos, L. M. Liz-Marzán, and C. Colliex, Nature Physics 3, 348 (2007).

61 Y. Francescato, V. Giannini, and S. A. Maier, ACS Nano 6, 1830 (2012).

62 C. L. G. Alzar, M. a. G. Martinez, and P. Nussenzveig, American Journal of Physics 70, 37 (2002).

63 M. A. Kats, N. Yu, P. Genevet, Z. Gaburro, and F. Capasso, Opt. Express 19, 21748 (2011).

64 M. Kociak and O. Stéphan, Chem. Soc. Rev. 43, 3865 (2014).

65 U. Hohenester, H. Ditlbacher, and J. R. Krenn, Phys. Rev. Lett. 103, 106801 (2009).

${ }^{66}$ F. J. G. d. Abajo and M. Kociak, New J. Phys. 10, 073035 (2008).

67 S. M. Collins and P. A. Midgley, Phys. Rev. B 87, 235432 (2013). 
68 E. Prodan, C. Radloff, N. J. Halas, and P. Nordlander, Science 302, 419 (2003).

${ }^{69}$ F. J. García de Abajo and J. Aizpurua, Phys. Rev. B 56, 15873 (1997).

70 A. E. Miroshnichenko, S. Flach, and Y. S. Kivshar, Rev. Mod. Phys. 82, 2257 (2010).

71 N. Yamamoto, K. Araya, and F. J. García de Abajo, Phys. Rev. B 64, 205419 (2001).

72 H. Liang, D. Rossouw, H. Zhao, S. K. Cushing, H. Shi, A. Korinek, H. Xu, F. Rosei, W. Wang, N. Wu, G. A. Botton, and D. Ma, Journal of the American Chemical Society 135, 9616 (2013).

73 L. Gu, W. Sigle, C. T. Koch, B. Ögüt, P. A. van Aken, N. Talebi, R. Vogelgesang, J. Mu, X. Wen, and J. Mao,
Phys. Rev. B 83, 195433 (2011).

74 B. Ögüt, R. Vogelgesang, W. Sigle, N. Talebi, C. T. Koch, and P. A. van Aken, ACS Nano 5, 6701 (2011).

75 M. Aeschlimann, M. Bauer, D. Bayer, T. Brixner, F. J. García de Abajo, W. Pfeiffer, M. Rohmer, C. Spindler, and F. Steeb, Nature 446, 301 (2007).

76 A. Yanai, M. Grajower, G. M. Lerman, M. Hentschel, H. Giessen, and U. Levy, ACS Nano 8, 4969 (2014).

77 C. Gruber, A. Trügler, A. Hohenau, U. Hohenester, and J. R. Krenn, Nano Lett. 13, 4257 (2013).

78 Q. Li, H. Wei, and H. Xu, Nano Lett. 14, 3358 (2014).

79 S. A. Mann and E. C. Garnett, Nano Lett. 13, 3173 (2013). 